ESAIM: M2AN 49 (2015) 1261-1283

DOI: $10.1051 / \mathrm{m} 2 \mathrm{an} / 2015010$
ESAIM: Mathematical Modelling and Numerical Analysis

www.esaim-m2an.org

\title{
A FINITE ELEMENT METHOD WITH SINGULARITY RECONSTRUCTION FOR FRACTIONAL BOUNDARY VALUE PROBLEMS
}

\author{
BANGTI JiN ${ }^{1}$ AND ZHI ZHOU ${ }^{2}$
}

\begin{abstract}
We consider a two-point boundary value problem involving a Riemann-Liouville fractional derivative of order $\alpha \in(1,2)$ in the leading term on the unit interval $(0,1)$. The standard Galerkin finite element method can only give a low-order convergence even if the source term is very smooth due to the presence of the singularity term $x^{\alpha-1}$ in the solution representation. In order to enhance the convergence, we develop a simple singularity reconstruction strategy by splitting the solution into a singular part and a regular part, where the former captures explicitly the singularity. We derive a new variational formulation for the regular part, and show that the Galerkin approximation of the regular part can achieve a better convergence order in the $L^{2}(0,1), H^{\alpha / 2}(0,1)$ and $L^{\infty}(0,1)$-norms than the standard Galerkin approach, with a convergence rate for the recovered singularity strength identical with the $L^{2}(0,1)$ error estimate. The reconstruction approach is very flexible in handling explicit singularity, and it is further extended to the case of a Neumann type boundary condition on the left end point, which involves a strong singularity $x^{\alpha-2}$. Extensive numerical results confirm the theoretical study and efficiency of the proposed approach.
\end{abstract}

Mathematics Subject Classification. 65M60, 65N30, 45J05.

Received April 13, 2014. Revised December 29, 2014.

Published online August 18, 2015.

\section{INTRODUCTION}

We consider the following fractional-order boundary value problem: find $u$ such that

$$
\begin{aligned}
-{ }_{0}^{R} D_{x}^{\alpha} u+q u & =f, \quad \text { in } D \equiv(0,1), \\
u(0)=u(1) & =0,
\end{aligned}
$$

where $\alpha \in(1,2)$ is the order of the derivative, and ${ }_{0}^{R} D_{x}^{\alpha}$ refers to the Riemann-Liouville fractional derivative of order $\alpha$ defined in (2.1) below. Here $f$ is a function in $L^{2}(D)$ or other suitable Sobolev space. The potential coefficient $q \in L^{\infty}(D)$ is a bounded measurable function. If the order $\alpha$ equals two, the fractional derivative ${ }_{0}^{R} D_{x}^{\alpha} u$ coincides with the usual second-order derivative $u^{\prime \prime}$ [13], and thus problem (1.1) generalizes the classical two-point boundary value problem.

Keywords and phrases. Finite element method, Riemann-Liouville derivative, fractional boundary value problem, error estimate, singularity reconstruction.

1 Department of Computer Science, University College London, Gower Street, London WC1E 6BT, UK.

bangti.jin@gmail.com

2 Department of Mathematics, Texas A\&M University, College Station, TX 77843-3368, USA. zzhou@math.tamu.edu 
The interest in the model (1.1) is largely motivated by anomalous diffusion, in which the mean square variance grows faster (superdiffusion) or slower (subdiffusion) than that in a Gaussian process. In recent years, a lot of works $[3,14]$ have shown that anomalous diffusion offers a superior fit to experimental data observed in transport phenomena in, e.g., viscoelastic materials, soil contamination, and underground water flow. The model (1.1) represents the steady state of one-dimensional asymmetric superdiffusion process, observed in magnetized plasma and geophysical flow $[5,6]$. It can be viewed as the macroscopic counterpart of Lévy flights, like Brownian motion for the classical diffusion equation; see [3] for a detailed derivation from the microscopic model and relevant physical explanations in the context of underground flow. Numerous experimental studies have demonstrated that it can capture accurately distinct features of superdiffusion processes.

The excellent modeling capabilities of the model (1.1) have generated considerable interest in deriving, analyzing and testing numerical methods for solving related initial boundary value problems. The finite difference scheme is predominant in existing studies, and it is usually based on a shifted Grünwald-Letnikov formula, which is first order accurate, for the Riemann-Liouville fractional derivative in space (see e.g. [17, 18]). In [7], a local discontinuous Galerkin method was developed. Shifted Grünwald-Letnikov formulas can be combined to achieve a second-order accuracy, under the assumption that the solution is sufficiently smooth [19]. However, the precise condition under which the solution is indeed smooth remains unknown. Very recently, we [12] developed a finite element method in space/the backward Euler (or Crank-Nicolson) method in time, based on the variational formulation developed in [11], and provided $L^{2}(D)$ error estimates for the approximation.

The mathematical study on the model (1.1) has just started to attract attention. First we note that the Riemann-Liouville fractional derivative operator is not the fractional power of the Laplace operator, and thus the well developed analytic theory on the fractional Laplacian does not apply to the model (1.1). Ervin and Roop [10] presented a first rigorous analysis of the weak formulation of problem (1.1), and an optimal $\widetilde{H}^{\alpha / 2}(D)$ error estimate for the Galerkin finite element method (FEM) was also provided under the assumption that the solution has full regularity, i.e., $\|u\|_{H^{\alpha}(D)} \leq C\|f\|_{L^{2}(D)}$, which however is generally not true [11] (see Sect. 2.1 below for the definition of the space $\left.\widetilde{H}^{s}(D)\right)$. Recently, Wang and Yang [20] developed a Petrov-Galerkin formulation of the model (1.1) with a variable coefficient, and analyzed its variational stability, but the discrete counterpart was not discussed. The model (1.1) was recently revisited in [11], and the case of a Caputo fractional derivative was also analyzed. Further, the proper variational formulations for both fractional derivatives were derived, and convergence rates of the Galerkin FEM approximations were established.

In [11], it was shown that the solution $u$ to (1.1) is generally only in the space $H^{\alpha-1+\beta}(D)$ with $\beta \in$ $[1-\alpha / 2,1 / 2)$ even if $f$ is much smoother than $L^{2}(D)$, due to the presence of the singular term $x^{\alpha-1}$ in the solution representation; see (2.3) below for details. This leads to a low-order convergence rate for the standard Galerkin FEM approximation based on continuous piecewise linear finite elements. There are several possible strategies for improving the convergence, e.g., adaptive refinement, solution space enrichment and singularity reconstruction. In this paper we opt for a simple strategy, inspired by the interesting work [4], that allows us to overcome this difficulty. Specifically, we split the solution $u$ into

$$
u=u^{r}+\mu u^{s},
$$

where $u^{r}$ denotes the regular part of the solution $u$ that has a better Sobolev regularity than $H^{\alpha-1+\beta}(D)$ with $\beta \in[1-\alpha / 2,1 / 2)$, and $u^{s}$ captures the leading singularity $x^{\alpha-1}$, with the scalar $\mu$ being the singularity strength. We derive a new variational formulation for the regular part $u^{r}$ and a reconstruction formula for the scalar $\mu$, and further, develop a stable finite element scheme for approximating the regular part $u^{r}$ and then reconstructing the singularity strength $\mu$. Theoretically, in Theorems 4.4 and 4.7, for the Galerkin approximation $u_{h}$, we derive error estimates in the $\widetilde{H}^{\alpha / 2}(D), L^{2}(D)$ and $L^{\infty}(D)$-norm, and the recovered singularity strength $\mu_{h}$ exhibits a convergence order identical with the $L^{2}(D)$ error estimate. Namely, for $f \in \widetilde{H}^{\gamma}(D), q \in \widetilde{H}^{\gamma}(D) \cap L^{\infty}(D)$, with $\beta \in[1-\alpha / 2,1 / 2)$ and $\ell(\beta, \gamma)=: \min (\alpha-1+\beta, \gamma)$, there holds

$$
\left\|u-u_{h}\right\|_{L^{2}(D)}+h^{\alpha / 2-1+\beta}\left\|u-u_{h}\right\|_{\widetilde{H}^{\alpha / 2}(D)} \leq C h^{\min (2, \alpha+\ell(\beta, \gamma))-1+\beta}\|f\|_{\widetilde{H}^{\gamma}(D)},
$$


which is higher than that for the classical approach, $c f$. Theorem 2.5 below. It is worth noting that all error estimates are expressed in terms of the right-hand side $f$ only. Numerically, the computational effort of the new approach is nearly identical with the classical one. Further, the singularity reconstruction approach is capable of resolving very strong singularity, which is highly nontrivial for other approaches, if not impossible at all. A strong solution singularity arises, for example, in the case of a Neumann boundary condition at the left end point, on which we shall further illustrate the flexibility of the proposed reconstruction approach.

The rest of the paper is organized as follows. In Section 2 we recall some important properties of fractional derivatives and integrals, and describe the variational formulation for the source problem (1.1) and the standard Galerkin FEM. We provide also a new $L^{\infty}(D)$-error estimate, besides the known $\widetilde{H}^{\alpha / 2}(D)$ and $L^{2}(D)$ error estimates. In Section 3, a novel variational formulation for the regular part $u^{r}$ of the solution and the reconstruction formula of the singularity strength $\mu$ are developed, and the stability of the formulation is established. The stability of the discrete variational formulation based on a Galerkin procedure is shown in Section 4, and the $\widetilde{H}^{\alpha / 2}(D), L^{2}(D)$ and $L^{\infty}(D)$ error estimates are also provided. Further, in Section 5, we extend the approach to the case of a Neumann boundary condition at the left end point, which involves a strong solution singularity $x^{\alpha-2}$, to illustrate its flexibility. Finally, numerical results are presented in Section 6 to confirm the convergence theory. Throughout, we use the notation $C$, with or without a subscript, to denote a generic constant, which can take different values at different occurrences, but it is always independent of the solution $u$ and the mesh size $h$.

\section{PRELiminaries}

In this part, we describe fundamentals of fractional calculus, the variational formulation for the source problem (1.1) and the Galerkin approximation.

\subsection{Fractional calculus}

We first briefly recall the Riemann-Liouville fractional derivative. For any $\beta>0$ with $n-1<\beta<n, n \in \mathbb{N}$, the left-sided Riemann-Liouville fractional derivative ${ }_{0}^{R} D_{x}^{\beta} u$ of order $\beta$ of a function $u \in H^{n}(D)$ is defined by ([13], p. 70):

$$
{ }_{0}^{R} D_{x}^{\beta} u=\frac{\mathrm{d}^{n}}{\mathrm{~d} x^{n}}\left({ }_{0} I_{x}^{n-\beta} u\right) .
$$

Here ${ }_{0} I_{x}^{\gamma}$ for $\gamma>0$ is the left-sided Riemann-Liouville fractional integral operator of order $\gamma$ defined by

$$
\left({ }_{0} I_{x}^{\gamma} f\right)(x)=\frac{1}{\Gamma(\gamma)} \int_{0}^{x}(x-t)^{\gamma-1} f(t) \mathrm{d} t,
$$

where $\Gamma(\cdot)$ is Euler's Gamma function defined by $\Gamma(x)=\int_{0}^{\infty} t^{x-1} \mathrm{e}^{-t} \mathrm{~d} t$. The right-sided versions of fractionalorder integral ${ }_{x} I_{1}^{\gamma}$ and derivative ${ }_{x}^{R} D_{1}^{\beta}$ are defined analogously by

$$
\left({ }_{x} I_{1}^{\gamma} f\right)(x)=\frac{1}{\Gamma(\gamma)} \int_{x}^{1}(t-x)^{\gamma-1} f(t) \mathrm{d} t \quad \text { and } \quad{ }_{x}^{R} D_{1}^{\beta} u=(-1)^{n} \frac{\mathrm{d}^{n}}{\mathrm{~d} x^{n}}\left({ }_{x} I_{1}^{n-\beta} u\right) .
$$

Now we introduce some function spaces. For any $\beta \geq 0$, we denote $H^{\beta}(D)$ to be the Sobolev space of order $\beta$ on the unit interval $D$, and $\widetilde{H}^{\beta}(D)$ to be the set of functions in $H^{\beta}(D)$ whose extension by zero to the real line $\mathbb{R}$ are in $H^{\beta}(\mathbb{R})$. Analogously, we define $\widetilde{H}_{L}^{\beta}(D)$ (respectively, $\widetilde{H}_{R}^{\beta}(D)$ ) to be the set of functions $u$ whose extension by zero, denoted by $\tilde{u}$, is in $H^{\beta}(-\infty, 1)$ (respectively, $H^{\beta}(0, \infty)$ ). For $u \in \widetilde{H}_{L}^{\beta}(D)$, we set $\|u\|_{\widetilde{H}_{L}^{\beta}(D)}:=\|\tilde{u}\|_{H^{\beta}(-\infty, 1)}$, and analogously the norm in $\widetilde{H}_{R}^{\beta}(D)$.

The following theorem collects their important properties. The proof of part (a) can be found in ([15], p. 46, Thm. 2.4) or ([13], p. 73, Lem. 2.3); and parts (b) and (c) can be found in ([11], Thms. 2.1 and 3.1). 
Theorem 2.1. The following statements hold.

(a) The integral operators ${ }_{0} I_{x}^{\beta}$ and ${ }_{x} I_{1}^{\beta}$ satisfy the semigroup property, i.e., for any $\beta, \gamma>0$

$$
{ }_{0} I_{x}^{\beta}\left({ }_{0} I_{x}^{\gamma} f\right)={ }_{0} I_{x}^{\beta+\gamma} f \quad \text { and } \quad{ }_{x} I_{1}^{\beta}\left({ }_{x} I_{1}^{\gamma} f\right)={ }_{x} I_{1}^{\beta+\gamma} f \quad \forall f \in L^{2}(D) .
$$

(b) The operators ${ }_{0}^{R} D_{x}^{\beta}: \widetilde{H}_{L}^{\beta}(D) \rightarrow L^{2}(D)$ and ${ }_{x}^{R} D_{1}^{\beta}: \widetilde{H}_{R}^{\beta}(D) \rightarrow L^{2}(D)$ are continuous.

(c) For any $s, \beta \geq 0$, the operator ${ }_{0} I_{x}^{\beta}$ is bounded from $\widetilde{H}_{L}^{s}(D)$ to $\widetilde{H}_{L}^{\beta+s}(D)$, and ${ }_{x} I_{1}^{\beta}$ is bounded from $\widetilde{H}_{R}^{s}(D)$ to $\widetilde{H}_{R}^{\beta+s}(D)$.

We shall also need an "algebraic" property of the space $\widetilde{H}^{s}(D), 0<s<1[11]$.

Lemma 2.2. Let $0<s \leq 1, s \neq 1 / 2$. Then for any $u \in H^{s}(D) \cap L^{\infty}(D)$ and $v \in \widetilde{H}^{s}(D) \cap L^{\infty}(D)$, uv $\in \widetilde{H}^{s}(D)$.

\subsection{Variational formulation}

Now we describe the variational formulation of problem (1.1), developed in [11]. We first consider the simplest source problem with a vanishing potential, i.e., $q \equiv 0$ :

$$
\begin{aligned}
-{ }_{0}^{R} D_{x}^{\alpha} u & =f \quad \text { in } D, \\
u(0)=u(1) & =0,
\end{aligned}
$$

with $f \in L^{2}(D)$. Note that ${ }_{0} I_{x}^{\alpha} f \in \widetilde{H}_{L}^{\alpha}(D)$ and thus by Theorem 2.1(b), ${ }_{0}^{R} D_{x}^{\alpha}\left({ }_{0} I_{x}^{\alpha} f\right) \in L^{2}(D)$ is well-defined. By Theorem 2.1(a) we have for all $f \in C_{0}^{\infty}(D)$

$$
{ }_{0}^{R} D_{x}^{\alpha}\left({ }_{0} I_{x}^{\alpha} f\right)=\left({ }_{0} I_{x}^{2-\alpha}\left({ }_{0} I_{x}^{\alpha} f\right)\right)^{\prime \prime}=\left(I^{2} f\right)^{\prime \prime}=f,
$$

and it is true for $f \in L^{2}(D)$ by a density argument; see also ([15], p. 44, Thm. 2.4) for discussions in the general case. Thus the solution $u$ of problem (2.2) has the explicit form:

$$
u(x)=-\left({ }_{0} I_{x}^{\alpha} f\right)(x)+\left({ }_{0} I_{x}^{\alpha} f\right)(1) x^{\alpha-1},
$$

by noting the relation ${ }_{0}^{R} D_{x}^{\alpha} x^{\alpha-1}=0$. The solution $u$ is only in $\widetilde{H}_{L}^{\alpha-1+\beta}(D)$ with $\beta \in[1-\alpha / 2,1 / 2)$ in general, even for a smooth source term $f$, due to the presence of the singular term $x^{\alpha-1}$.

The variational formulation of problem $(2.2)$ is given by $[10,11]$ : find $u \in V \equiv \widetilde{H}^{\alpha / 2}(D)$ such that

$$
A(u, v)=(f, v) \quad \forall v \in V,
$$

with the bilinear form $A(\cdot, \cdot)$ defined on $V \times V$ by

$$
A(u, v)=-\left({ }_{0}^{R} D_{x}^{\alpha / 2} u,{ }_{x}^{R} D_{1}^{\alpha / 2} v\right) \quad \forall u, v \in V .
$$

It is known that the bilinear form $A(\cdot, \cdot)$ is coercive on the space $V$ (see [10], Lem. 3.1, [11], Lem 4.2), i.e., there is a constant $C_{0}$ such that for all $u \in V$

$$
A(u, u) \geq C_{0}\|u\|_{V}^{2}
$$

and it is continuous on $V$, i.e., for all $u, v \in V$

$$
|A(u, v)| \leq C_{1}\|u\|_{V}\|v\|_{V} .
$$

We now turn to the general case $q \neq 0$ and define

$$
a(u, v)=A(u, v)+(q u, v) .
$$

Then the variational formulation for problem (1.1) is given by: to find $u \in V$ such that

$$
a(u, v)=(f, v) \quad \forall v \in V .
$$

To study the bilinear form $a(\cdot, \cdot)$, we make the following assumption. The assumption holds automatically for the case $q \geq 0$, in view of the coercivity of the bilinear form $A(\cdot, \cdot)$ on the space $\widetilde{H}^{\alpha / 2}(D)([11]$, Lem. 4.2). 
Assumption 2.3. Let the bilinear form $a(u, v)$ with $u, v \in V$ satisfy

(a) The problem of finding $u \in V$ such that $a(u, v)=0$ for all $v \in V$ has only the trivial solution $u \equiv 0$.

$\left(\mathrm{a}^{*}\right)$ The problem of finding $v \in V$ such that $a(u, v)=0$ for all $u \in V$ has only the trivial solution $v \equiv 0$.

Under Assumption 2.3, there exists a unique solution $u \in V$ to (2.8) ([11], Thm. 4.3). In fact the variational solution is a strong solution. To see this, we consider the problem

$$
-{ }_{0}^{R} D_{x}^{\alpha} u=f-q u .
$$

A strong solution is given by (2.3) with a right hand side $\widetilde{f}=f-q u$. It satisfies the variational problem (2.8) and hence coincides with the unique variational solution. Further, it satisfies the following regularity

$$
\|u\|_{\widetilde{H}_{L}^{\alpha-1+\beta}(D)} \leq C\|f\|_{L^{2}(D)},
$$

for any $\beta \in[1-\alpha / 2,1 / 2)$. Thus the global regularity of the solution $u$ does not improve with the regularity of the source term $f$, due to the inherent presence of the term $x^{\alpha-1}$ in the solution representation (2.3).

\subsection{Standard Galerkin FEM}

Now we describe the standard finite element approximation based on a uniform partition of the interval $D$. Let $h=1 / m$ be the mesh size with $m>1$ being a positive integer, and the nodes $x_{j}=j h, j=0, \ldots, m$. We then define $V_{h}$ to be the continuous piecewise linear finite element space, i.e.,

$$
V_{h}=\left\{\chi \in C_{0}(\bar{D}): \chi \text { is linear over }\left[x_{j}, x_{j+1}\right], j=0, \ldots, m-1\right\} .
$$

It is well-known that the space $V_{h}$ has the following approximation properties.

Lemma 2.4. If $u \in H^{\gamma}(D) \cap \widetilde{H}^{\alpha / 2}(D)$ with $\alpha / 2 \leq \gamma \leq 2$, then

$$
\inf _{v \in V_{h}}\|u-v\|_{H^{\alpha / 2}(D)} \leq c h^{\gamma-\alpha / 2}\|u\|_{H^{\gamma}(D)} .
$$

The Galerkin FEM problem is to find $u_{h} \in V_{h}$ such that

$$
a\left(u_{h}, v\right)=(f, v) \quad \forall v \in V_{h} .
$$

The discrete approximation $u_{h}$ exists and is unique for small $h$, and further, it satisfies the following error estimates ([11], Thm. 5.2). The result has the flavor of Mikhlin's theorem in the sense of "asymptotic stability".

Theorem 2.5. Let Assumption 2.3 hold, $f \in L^{2}(D)$, and $q \in L^{\infty}(D)$. Then there is an $h_{0}>0$ such that for all $h \leq h_{0}$, problem (2.11) has a unique solution $u_{h} \in V_{h}$ and it satisfies for any $\beta \in[1-\alpha / 2,1 / 2)$

$$
\left\|u-u_{h}\right\|_{L^{2}(D)}+h^{\alpha / 2-1+\beta}\left\|u-u_{h}\right\|_{H^{\alpha / 2}(D)} \leq C h^{\alpha-2+2 \beta}\|f\|_{L^{2}(D)} .
$$

Remark 2.6. Due to the inherent presence of the singular term $x^{\alpha-1}$, the solution $u$ has rather low global regularity, especially for $\alpha$ close to unity, even if the source term $f$ is smooth. Hence, the convergence rate of the Galerkin approximation $u_{h}$ based on the formulation (2.11) is slow: for $\alpha$ close to unity, the theoretical rate is close to zero. This necessitates the development of new techniques with enhanced convergence rates.

Next we derive a new $L^{\infty}(D)$-error estimate for the Galerkin approximation $u_{h}$. To this end, we first recall Green's function to the adjoint problem of (1.1), i.e., for all $x \in D$

$$
\begin{aligned}
-{ }_{y}^{R} D_{1}^{\alpha} G(x, y)+q(y) G(x, y) & =\delta_{x}(y), \quad y \in D, \\
G(x, 0)=G(x, 1) & =0 .
\end{aligned}
$$


For the case $q \equiv 0, G(x, y)$ is available in closed form [2]. It follows from the solution representation (2.3) that $G(x, y)$ is given explicitly by

$$
G(x, y)= \begin{cases}\frac{(1-y)^{\alpha-1} x^{\alpha-1}}{\Gamma(\alpha)}, & 0 \leq x \leq y \leq 1 \\ \frac{(1-y)^{\alpha-1} x^{\alpha-1}-(x-y)^{\alpha-1}}{\Gamma(\alpha)}, & 0 \leq y \leq x \leq 1 .\end{cases}
$$

We note that $G(x, \cdot) \in \widetilde{H}^{\alpha-1+\beta}(D)$ with $\beta \in[1-\alpha / 2,1 / 2)$. In fact, the fractional integral ${ }_{y} I_{1}^{\alpha} \delta_{x}(y)$ of the Dirac-delta function $\delta_{x}(y)$ is given by

$$
\left({ }_{y} I_{1}^{\alpha} \delta_{x}\right)(y)= \begin{cases}0, & 0 \leq x \leq y \leq 1 \\ \frac{(x-y)^{\alpha-1}}{\Gamma(\alpha)}, & 0 \leq y \leq x \leq 1\end{cases}
$$

which lies in $\widetilde{H}^{\alpha-1+\beta}(D)$ with $\beta \in[1-\alpha / 2,1 / 2)$. In the case of a general potential $q \neq 0$, the weak formulation is to find $G(x, \cdot) \in V$ such that

$$
a(v, G(x, \cdot))=\left\langle v, \delta_{x}\right\rangle=v(x) \quad \forall v \in V .
$$

By Sobolev embedding theorem [1], $\delta_{x} \in V^{*}$, the dual space of $V$, and thus the existence and uniqueness of $G(x, y) \in \widetilde{H}^{\alpha / 2}(D)$ follows directly from the stability of the variational formulation. Moreover, it satisfies the differential equation

$$
-{ }_{y}^{R} D_{1}^{\alpha} G(x, y)=\delta_{x}(y)-q(y) G(x, y), \quad y \in D .
$$

Then the facts that $q(\cdot) G(x, \cdot) \in L^{2}(D)$ and ${ }_{y} I_{1}^{\alpha} \delta_{x} \in \widetilde{H}_{R}^{\alpha-1+\beta}(D)$ lead to the desired regularity $G(x, \cdot) \in$ $\widetilde{H}_{R}^{\alpha-1+\beta}(D)$ with $\beta \in[1-\alpha / 2,1 / 2)$.

Now we can state an $L^{\infty}(D)$-error estimate of the Galerkin approximation $u_{h}$.

Theorem 2.7. Let $q \in L^{\infty}(D)$, Assumption 2.3 hold, and $f \in L^{2}(D)$. Then there is an $h_{0}>0$ such that for all $h \leq h_{0}$, the solution $u_{h}$ to problem (2.11) satisfies for any $\beta \in[1-\alpha / 2,1 / 2)$

$$
\left\|u-u_{h}\right\|_{L^{\infty}(D)} \leq C h^{\alpha-2+2 \beta}\|f\|_{L^{2}(D)} .
$$

Proof. Using the weak formulation (2.15) of $G(x, y)$ and the Galerkin orthogonality, we have for any $w_{h} \in V_{h}$

$$
\left(u-u_{h}\right)(x)=a\left(u-u_{h}, G(x, \cdot)\right)=a\left(u-u_{h}, G(x, \cdot)-w_{h}\right) .
$$

Then applying (2.7), Lemma 2.4 and Theorem 2.5, we obtain for any $\beta \in[1-\alpha / 2,1 / 2)$

$$
\begin{aligned}
\left|\left(u-u_{h}\right)(x)\right| & \leq C\left\|u-u_{h}\right\|_{V} \inf _{w_{h} \in V_{h}}\left\|G(x, \cdot)-w_{h}\right\|_{V} \\
& \leq C h^{\alpha-2+2 \beta}\|u\|_{\widetilde{H}_{L}^{\alpha-1+\beta}(D)} \leq C h^{\alpha-2+2 \beta}\|f\|_{L^{2}(D)} .
\end{aligned}
$$

Remark 2.8. It is well-known that for the standard second-order two-point boundary value problem, the Galerkin approximation $u_{h}$ exhibits superconvergence at the nodes due to the piecewise smoothness of Green's function [8]. For the fractional counterpart, the Green's function is no longer piecewise smooth: it is only in $\widetilde{H}_{L}^{\alpha-1+\beta}(D)$ with $\beta \in[1-\alpha / 2,1 / 2)$ even for $q=0$. Our numerical experiments in Section 6 indicate that the $L^{\infty}(D)$-error estimate in Theorem 2.7 is sharp.

\section{A NEW VARiational FORMUlation AND WELl-POSEDNESS}

Now we develop a new approach for the source problem (1.1) based on singularity splitting, which is inspired by the interesting work [4], developed for the Poisson equation on the L-shaped domain. We shall derive a new variational formulation for the regular part, and establish its stability and the regularity pickup. 


\subsection{Derivation of the new approach}

The new approach is based on splitting the solution $u$ to problem (1.1) into a regular part $u^{r}$ and a singular part involving $x^{\alpha-1}$ :

$$
u(x)=u^{r}+\mu\left(x^{\alpha-1}-x^{2}\right) .
$$

In the splitting, the choice of $x^{2}$ is arbitrary. We shall assume

$$
{ }_{0} I_{x}^{\alpha}\left(q\left(x^{\alpha-1}-x^{2}\right)\right)(1) \neq-1 .
$$

If this condition does not hold, we may replace the choice $x^{2}$ by any other function $v$ in the space $\widetilde{H}_{L}^{s}(D), s \geq 2$, with $v(1)=1$, such that ${ }_{0} I_{x}^{\alpha}\left(q\left(x^{\alpha-1}-v\right)\right)(1) \neq-1$. Now we develop a new variational formulation to uniquely determine the regular part $u^{r}$. First, we deduce from (2.3) and (2.9) that the solution $u$ can be split into

$$
\begin{aligned}
u(x)= & -{ }_{0} I_{x}^{\alpha}(f-q u)(x)+{ }_{0} I_{x}^{\alpha}(f-q u)(1) x^{\alpha-1} \\
= & -{ }_{0} I_{x}^{\alpha}(f-q u)(x)+{ }_{0} I_{x}^{\alpha}(f-q u)(1) x^{2} \\
& +{ }_{0} I_{x}^{\alpha}(f-q u)(1)\left(x^{\alpha-1}-x^{2}\right) .
\end{aligned}
$$

Let $\mu={ }_{0} I_{x}^{\alpha}(f-q u)(1)$. We can set the regular part $u^{r}$ and singular part $u^{s}$ to

$$
u^{r}(x)=-{ }_{0} I_{x}^{\alpha}(f-q u)(x)+\mu x^{2} \quad \text { and } \quad u^{s}(x)=x^{\alpha-1}-x^{2},
$$

respectively. Then obviously,

$$
u(x)=u^{r}(x)+\mu u^{s}(x) .
$$

To construct a variational problem for the regular part $u^{r}$, we first derive a reconstruction formula for the singularity strength $\mu$ in terms of $u^{r}$. By the definition of $\mu$ and the regular part $u^{r}, c f$. (3.1), we have

$$
\mu={ }_{0} I_{x}^{\alpha}\left(f-q\left(u^{r}+\mu\left(x^{\alpha-1}-x^{2}\right)\right)\right)(1) .
$$

By rearranging terms, $\mu$ can be written as

$$
\mu=c_{0}\left({ }_{0} I_{x}^{\alpha}\left(f-q u^{r}\right)\right)(1)
$$

where the constant $c_{0}$ is defined by

$$
c_{0}=\frac{1}{1+{ }_{0} I_{x}^{\alpha}\left(q\left(x^{\alpha-1}-x^{2}\right)\right)(1)} .
$$

Hence the solution $u$ of problem (1.1) can be split into

$$
u=u^{r}+c_{0}\left({ }_{0} I_{x}^{\alpha}\left(f-q u^{r}\right)\right)(1)\left(x^{\alpha-1}-x^{2}\right) .
$$

Upon substituting it back into (1.1) and setting

$$
c_{1}(x)={ }_{0}^{R} D_{x}^{\alpha}\left(x^{\alpha-1}-x^{2}\right)=-\frac{2}{\Gamma(3-\alpha)} x^{2-\alpha},
$$

we arrive at the following fractional integro-differential problem for the regular part $u^{r}$

$$
\begin{gathered}
-{ }_{0}^{R} D_{x}^{\alpha} u^{r}+q u^{r}+Q\left({ }_{0} I_{x}^{\alpha}\left(q u^{r}\right)\right)(1)=\widetilde{f} \quad \text { in } D, \\
u^{r}(0)=u^{r}(1)=0,
\end{gathered}
$$

where the functions $Q(x)$ and $\widetilde{f}(x)$ are defined respectively by

$$
\begin{aligned}
& Q(x)=c_{0} c_{1}(x)-c_{0} q(x)\left(x^{\alpha-1}-x^{2}\right) \in L^{\infty}(D), \\
& \widetilde{f}(x)=f(x)+c_{0} c_{1}(x)\left({ }_{0} I_{x}^{\alpha} f\right)(1)-c_{0}\left({ }_{0} I_{x}^{\alpha} f\right)(1) q(x)\left(x^{\alpha-1}-x^{2}\right) \in L^{2}(D) .
\end{aligned}
$$




\subsection{A new variational formulation and its stability}

For problem (3.4) for the regular part $u^{r}$, we introduce the following bilinear form

$$
a_{r}(u, v)=A(u, v)+b(u, v) \quad \forall u, v \in V,
$$

with the form $b(\cdot, \cdot)$ given by

$$
b(u, v)=(q u, v)+{ }_{0} I_{x}^{\alpha}(q u)(1)(Q, v) \quad \forall u, v \in V .
$$

Using Theorem 2.1(c), $q \in L^{\infty}(D)$, and Sobolev embedding theorem [1], we deduce

$$
\begin{aligned}
|b(u, v)| & \leq{ }_{0} I_{x}^{\alpha}(q u)(1)\|(Q, v) \mid+\| q u\left\|_{L^{2}(D)}\right\| v \|_{L^{2}(D)} \\
& \leq C\left\|_{0} I_{x}^{\alpha}(q u)\right\|_{\widetilde{H}_{L}^{\alpha}(D)}\|Q\|_{L^{2}(D)}\|v\|_{L^{2}(D)}+C\|u\|_{L^{2}(D)}\|v\|_{L^{2}(D)} \\
& \leq C\|u\|_{L^{2}(D)}\|v\|_{L^{2}(D)} .
\end{aligned}
$$

That is, $b(\cdot, \cdot)$ is continuous on $L^{2}(D) \times L^{2}(D)$. Thus the bilinear form $a_{r}(\cdot, \cdot)$ is continuous on $V \times V$, i.e., there exists a constant $C>0$ such that

$$
a_{r}(u, v) \leq C\|u\|_{V}\|v\|_{V} \quad \forall u, v \in V .
$$

The variational problem for the regular part $u^{r}$ reads: find $u^{r} \in V$ satisfying

$$
a_{r}\left(u^{r}, v\right)=(\tilde{f}, v) \quad \forall v \in V .
$$

Once the regular part $u^{r}$ is determined, the singularity strength $\mu$ can be reconstructed using formula (3.2). Finally the solution $u$ of the source problem (1.1) has the following representation:

$$
u=u^{r}+\mu\left(x^{\alpha-1}-x^{2}\right)
$$

Now we turn to the well-posedness of the variational formulation (3.10). In case of $q=0$, the bilinear form $a_{r}(\cdot, \cdot)$ is identical with $a(\cdot, \cdot)$. Thus the well-posednees of problem $(3.10)$ follows directly from the continuity and coercivity of the bilinear form. It suffices to consider the case $q \neq 0$. To this end, we make the following uniqueness assumption on the bilinear form $a_{r}(\cdot, \cdot)$.

Assumption 3.1. Let the bilinear form $a_{r}(u, v)$ with $u, v \in V$ satisfy

(a) The problem of finding $u \in V$ such that $a_{r}(u, v)=0$ for all $v \in V$ has only the trivial solution $u \equiv 0$.

$\left(\mathrm{a}^{*}\right)$ The problem of finding $v \in V$ such that $a_{r}(u, v)=0$ for all $u \in V$ has only the trivial solution $v \equiv 0$.

The next result shows that Assumption 2.3 implies Assumption 3.1(a). However, the connection between Assumptions 2.3 and $3.1\left(\mathrm{a}^{*}\right)$ is still unclear.

Lemma 3.2. Assumption 2.3 implies Assumption 3.1(a).

Proof. If $\widetilde{f} \equiv 0$, then by the definition of $\widetilde{f}$ in (3.5) we have

$$
f(x)=-c_{0} c_{1}(x)\left({ }_{0} I_{x}^{\alpha} f\right)(1)+c_{0}\left(\left({ }_{0} I_{x}^{\alpha} f\right)(1) q(x)\left(x^{\alpha-1}-x^{2}\right)\right) .
$$

We observe that $f$ has an explicit representation for any $c \in \mathbb{R}$

$$
f=c\left(-c_{1}(x)+q(x)\left(x^{\alpha-1}-x^{2}\right)\right) .
$$

Now suppose that there exists a $\chi \in V, \chi \neq 0$, such that $a_{r}(\chi, v)=0$ for all $v \in V$. Then by construction

$$
u=\chi-c_{0}\left({ }_{0} I_{x}^{\alpha}(q \chi)\right)(1)\left(x^{\alpha-1}-x^{2}\right)
$$


is a solution of (1.1) with the right hand side $f$. Meanwhile, Assumption 2.3 implies that problem (1.1) has a unique solution

$$
u=c\left(x^{\alpha-1}-x^{2}\right)
$$

Then comparing these two solution representations yields that

$$
\chi=c^{\prime}\left(x^{\alpha-1}-x^{2}\right) .
$$

where $c^{\prime} \neq 0$. Now using the fact that

$$
A\left(x^{\alpha-1}-x^{2}, v\right)=\left(-{ }_{0}^{R} D_{x}^{\alpha}\left(x^{\alpha-1}-x^{2}\right), v\right) \quad \forall v \in V,
$$

we deduce that $\chi$ satisfies (3.4). Then plugging (3.12) into (3.4) and simple computation yield

$$
c_{0}{ }_{0} I_{x}^{\alpha}\left(q\left(x^{\alpha-1}-x^{2}\right)\right)(1)=1
$$

which is contradictory to the definition of $c_{0}$.

Under Assumption 3.1, the variational formulation (3.10) is stable.

Theorem 3.3. Let Assumption 3.1 hold and $q \in L^{\infty}(D)$. Then for any $F \in V^{*}$, there exists a unique solution $u \in V$ to

$$
a_{r}(u, v)=\langle F, v\rangle \quad \forall v \in V
$$

Proof. The proof is based on Petree-Tartar Lemma ([9], p. 469, Lem. A.38). We define two operators $S \in$ $\mathcal{L}\left(V ; V^{*}\right)$ and $T \in \mathcal{L}\left(V ; V^{*}\right)$ by

$$
\langle S u, v\rangle=a_{r}(u, v) \quad \text { and } \quad(T u, v)=-b(u, v),
$$

respectively. By Assumption 3.1(a), the operator $S$ is injective. Further,

$$
\begin{aligned}
(T u)(x) & =-\int_{0}^{1} \frac{Q(x) q(y)(1-y)^{\alpha-1}}{\Gamma(\alpha)} u(y) \mathrm{d} y-q(x) u(x) \\
& =:\left(T_{1} u\right)(x)+\left(T_{2} u\right)(x) .
\end{aligned}
$$

Now $Q \in L^{\infty}(D)$ implies that $T_{1}$ is a Hilbert-Schmidt operator and hence compact ([21], p. 277, example 2). Further, $T_{2}$ is also compact in view of the assumption $q \in L^{\infty}(D)$ and the compactness of the embedding from $V$ into $L^{2}(D)$. Thus $T$ is a compact operator from $V$ to $L^{2}(D)$. By the coercivity (2.6) and continuity (2.7) of the bilinear form $A(\cdot, \cdot)$, we obtain

$$
C_{0}\|u\|_{V}^{2} \leq A(u, u)=a_{r}(u, u)-b(u, u) \leq C\left(\|T u\|_{V^{*}}+\|S u\|_{V^{*}}\right)\|u\|_{V},
$$

Now the Petree-Tartar lemma immediately implies that the image of the operator $S$ is closed; equivalently, there exists a constant $\delta>0$ satisfying

$$
\delta\|u\|_{V} \leq \sup _{v \in V} \frac{a_{r}(u, v)}{\|v\|_{V}}
$$

This together with Assumption 3.1( $\left.\mathrm{a}^{*}\right)$ yields the unique existence of a solution $u \in U$ to problem (3.13).

Now we state an improved regularity result for $\langle F, v\rangle=(f, v)$, for some $f \in \widetilde{H}^{\gamma}(D), 0 \leq \gamma \leq 1, \gamma \neq 1 / 2$.

Theorem 3.4. Let Assumption 3.1 hold, $q \in \widetilde{H}^{\gamma}(D) \cap L^{\infty}(D)$ and $f \in \widetilde{H}^{\gamma}(D)$ with $0 \leq \gamma \leq 1, \gamma \neq 1 / 2$. There exists a unique solution $u^{r} \in \widetilde{H}^{\alpha / 2}(D)$ to problem (3.10) and further, for any $\beta \in[1-\alpha / 2,1 / 2)$, with $\ell(\beta, \gamma)=: \min (\alpha-1+\beta, \gamma)$, it satisfies

$$
\left\|u^{r}\right\|_{H^{\alpha+\ell(\beta, \gamma)}(D)} \leq C\|f\|_{\widetilde{H}^{\gamma}(D)} .
$$


Proof. The unique existence of a solution $u^{r} \in V$ follows from Theorem 3.3. Hence, it suffices to show the stability estimate. By its construction, the solution $u^{r}$ is of the form (3.1). By $q \in \widetilde{H}^{\gamma}(D) \cap L^{\infty}(D)$ and $u \in \widetilde{H}_{L}^{\alpha-1+\beta}(D)$, and by Lemma 2.2 , we deduce $q u \in \widetilde{H}_{L}^{\min (\gamma, \alpha-1+\beta)}(D)$. Now with Theorem 2.1(c), ${ }_{0} I_{x}^{\alpha}(f-q u) \in$ $\widetilde{H}_{L}^{\alpha+\gamma(\beta)}(D)$.

Corollary 3.5. Let Assumption 3.1 hold, $u^{r}$ be the solution of (3.10), and the singularity strength $\mu$ be defined by (3.2). Then $u=u^{r}+\mu\left(x^{\alpha-1}-x^{2}\right)$ is the solution of (1.1).

\subsection{Adjoint problem}

To derive error estimates for the Galerkin approximation $u_{h}^{r}$ of the regular part $u^{r}$ in Section 4 below, it is useful to consider the adjoint problem to (3.10). For $F \in V^{*}$, the dual problem is to find $w \in V$ such that

$$
a_{r}(v, w)=\langle v, F\rangle \quad \forall v \in V .
$$

In the case of $\langle v, F\rangle=(v, f)$ for some $f \in L^{2}(D)$, the strong form reads

$$
\begin{gathered}
-{ }_{x}^{R} D_{1}^{\alpha} w+q w+\frac{(1-x)^{\alpha-1} q}{\Gamma(\alpha)}(Q, w)=f \quad \text { in } D, \\
w(0)=w(1)=0 .
\end{gathered}
$$

For the case $q \equiv 0$, the solution $w$ to (3.16) is given by

$$
w=-\left({ }_{x} I_{1}^{\alpha} f\right)(x)+\left({ }_{x} I_{1}^{\alpha} f\right)(0)(1-x)^{\alpha-1},
$$

and hence, $w \in \widetilde{H}_{R}^{\alpha-1+\beta}(D) \cap \widetilde{H}^{\alpha / 2}(D)$. The case $q \neq 0$ is treated in the following theorem.

Theorem 3.6. Let $q \in L^{\infty}(D)$, and Assumption 3.1 hold. Then with a right hand side $\langle v, F\rangle=(v, f)$ for some $f \in L^{2}(D)$, the solution $w$ to problem (3.15) belongs to $\widetilde{H}_{R}^{\alpha-1+\beta}(D) \cap \widetilde{H}^{\alpha / 2}(D)$ and satisfies for any $\beta \in[1-\alpha / 2,1 / 2)$

$$
\|w\|_{\widetilde{H}_{R}^{\alpha-1+\beta}(D)} \leq C\|f\|_{L^{2}(D)}
$$

Proof. The strong problem for $w$ can be rewritten as

$$
-{ }_{x}^{R} D_{1}^{\alpha} w(x)=\bar{f}:=-q(x) w(x)-\frac{(1-x)^{\alpha-1} q(x)}{\Gamma(\alpha)}(Q, w)+f(x), \quad x \in D,
$$

with the boundary condition $w(0)=w(1)=0$. Since $q \in L^{\infty}(D)$ and $w \in \widetilde{H}^{\alpha / 2}(D)$, there holds $q w \in L^{2}(D)$, and the source term $\bar{f} \in L^{2}(D)$. The assertion follows from the representation (3.17) and Theorem 2.1(c).

Remark 3.7. In general, the best possible regularity of the solution $w$ to the adjoint problem (3.15) lies in $\widetilde{H}_{R}^{\alpha-1+\beta}(D)$ for $\beta \in[1-\alpha / 2,1)$ due to the presence of the singular term $(1-x)^{\alpha-1}$. The only possibility of full regularity is the case $\left({ }_{x} I_{1}^{\alpha} f\right)(0)=0$ (for $\left.q=0\right)$.

\section{Galerkin FEM FOR THE NEW FORMUlation}

Now we apply the variational formulation developed in Section 3 to the numerical approximation of problem (1.1). We shall analyze the stability of the discrete variational formulation, and derive error estimates for the discrete approximations. 


\subsection{Galerkin FEM}

Based on the variational formulation (3.10), we can develop a new Galerkin FEM for problem (1.1) with enhanced convergence rates. First, we approximate the regular part $u^{r}$ using a Galerkin procedure over the continuous piecewise linear finite element space $V_{h}$. The choice of piecewise linear elements is motivated by the following empirical observation: due to the presence of the potential term $q$, the solution $u$ to the fractional model (1.1) can contain a hierarchy of weak singularities, apart from the leading one $x^{\alpha-1}$, and thus higher-order elements are not expected to be efficient in general. The discrete counterpart of (3.10) is to find $u_{h}^{r} \in V_{h}$ such that

$$
a_{r}\left(u_{h}^{r}, v\right)=(\tilde{f}, v) \quad \forall v \in V_{h},
$$

where the bilinear form $a_{r}(\cdot, \cdot)$ and the source term $\tilde{f} \in L^{2}(D)$ are defined in (3.10) and (3.5), respectively. Then we reconstruct a finite element approximation $\mu_{h}$ to the strength $\mu$ of the singular part $u^{s}$ by

$$
\mu_{h}=c_{0}\left({ }_{0} I_{x}^{\alpha}\left(f-q u_{h}^{r}\right)\right)(1),
$$

where the constant $c_{0}$ is defined in (3.3). Last, we construct an approximate solution $u_{h}$ to (1.1) by

$$
u_{h}=u_{h}^{r}+\mu_{h}\left(x^{\alpha-1}-x^{2}\right) .
$$

In order to derive an error estimate, we first establish the well-posedness of problem (4.1). To this end, we need the (adjoint) Ritz projection $R_{h}: V \rightarrow V_{h}$ defined by

$$
A\left(v, R_{h} u\right)=A(v, u) \quad \forall u \in V, v \in V_{h} .
$$

Then Céa's lemma and finite element duality imply that there hold for any $\beta \in[1-\alpha / 2,1 / 2)$

$$
\begin{aligned}
\left\|R_{h} u\right\|_{V} & \leq C\|u\|_{V} & & \forall u \in V, \\
\left\|u-R_{h} u\right\|_{L^{2}(D)} & \leq C h^{\alpha / 2-1+\beta}\|u\|_{V} & & \forall u \in V .
\end{aligned}
$$

Note that the $L^{2}(D)$ error estimate of the adjoint Ritz projection $R_{h}$ is suboptimal, due to the low global regularity of the adjoint solution.

Next, we show the stability of the discrete variational problem (4.1).

Theorem 4.1. Let Assumption 3.1 hold, $f \in L^{2}(D)$, and $q \in L^{\infty}(D)$. Then there is an $h_{0}>0$ such that for all $h \leq h_{0}$ the finite element problem: finding $u_{h}^{r} \in V_{h}$ such that

$$
a_{r}\left(u_{h}^{r}, v\right)=(f, v) \quad \forall v \in V_{h}
$$

has a unique solution.

Proof. The unique existence of the discrete solution $u_{h}^{r} \in V_{h}$ when $q \equiv 0$ is a direct consequence of the coercivity and continuity of the bilinear form $A(\cdot, \cdot)$ on the space $V \times V, c f .(2.6)$ and (2.7), and Lax-Milgram theorem. For the case $q \neq 0$, we show the inf-sup condition for the bilinear form $a_{r}(\cdot, \cdot)$ on the space $V_{h} \times V_{h}$ using a kick-back argument analogous to Schatz [16].

By the inf-sup condition (3.14), we have for $z_{h} \in V_{h} \subset V \equiv \widetilde{H}^{\alpha / 2}(D)$

$$
\delta\left\|z_{h}\right\|_{V} \leq \sup _{v \in V} \frac{a_{r}\left(z_{h}, v\right)}{\|v\|_{V}} \leq \sup _{v \in V} \frac{a_{r}\left(z_{h}, v-R_{h} v\right)}{\|v\|_{V}}+\sup _{v \in V} \frac{a_{r}\left(z_{h}, R_{h} v\right)}{\|v\|_{V}} .
$$

In view of Galerkin orthogonality of (adjoint) Ritz-projection $R_{h}$ and the continuity of bilinear form $b(\cdot, \cdot)$ in (3.8), we have

$$
\sup _{v \in V} \frac{a_{r}\left(z_{h}, v-R_{h} v\right)}{\|v\|_{V}}=\sup _{v \in V} \frac{b\left(z_{h}, v-R_{h} v\right)}{\|v\|_{V}} \leq C \sup _{v \in V} \frac{\left\|z_{h}\right\|_{L^{2}(D)}\left\|v-R_{h} v\right\|_{L^{2}(D)}}{\|v\|_{V}} .
$$


For the first term in (4.7), using (4.5), we obtain for all $\beta \in[1-\alpha / 2,1 / 2)$

$$
\sup _{v \in V} \frac{a_{r}\left(z_{h}, v-R_{h} v\right)}{\|v\|_{V}} \leq C h^{\alpha / 2-1+\beta}\left\|z_{h}\right\|_{L^{2}(D)} \leq C_{1} h^{\alpha / 2-1+\beta}\left\|z_{h}\right\|_{V} .
$$

Now by the stability of $R_{h}$ in (4.5), we deduce the following estimate for the second term in (4.7)

$$
\sup _{v \in V} \frac{a_{r}\left(z_{h}, R_{h} v\right)}{\|v\|_{V}} \leq C \sup _{v \in V} \frac{a_{r}\left(z_{h}, R_{h} v\right)}{\left\|R_{h} v\right\|_{V}} \leq C_{2} \sup _{v \in V_{h}} \frac{a_{r}\left(z_{h}, v\right)}{\|v\|_{V}}
$$

By choosing $h_{0}$ such that $C_{1} h_{0}^{\alpha / 2-1+\beta}=\delta / 2$, we arrive at the desired discrete inf-sup condition on $V_{h} \times V_{h}$ :

$$
\frac{\delta}{2}\left\|z_{h}\right\|_{V} \leq C_{2} \sup _{v \in V_{h}} \frac{a_{r}\left(z_{h}, v\right)}{\|v\|_{V}} \text { for } \quad h \leq h_{0} .
$$

This shows that there is a unique solution of (4.1).

Before providing error estimates, we note that the matrix analogue of the bilinear form $A(\cdot, \cdot)$ is of lowerHessenberg form, and that for the integral part is of rank one. Hence, the matrix for the bilinear form $a_{r}(\cdot, \cdot)$ is a rank-one perturbation of a lower-Hessenberg matrix. Further, the stiffness matrix for the leading term $-\left({ }_{0}^{R} D_{x}^{\alpha / 2} \cdot,{ }_{x}^{R} D_{1}^{\alpha / 2} \cdot\right)$ is Toeplitz when the mesh is uniform, which can be verified directly. This represents a very important structural property that can be exploited in forming the stiffness matrix, efficient storage and fast iterative solution of the resulting linear system, by e.g., GMRES.

\subsection{Error estimates}

Next we establish error estimates for the Galerkin approximation $u_{h}$ given by (4.3). We first analyze the $L^{2}(D)$ - and $\widetilde{H}^{\alpha / 2}(D)$-norm of the error $u^{r}-u_{h}^{r}$ for the regular part $u^{r}$.

Theorem 4.2. Let Assumption 3.1 hold, $f \in \widetilde{H}^{\gamma}(D)$, and $q \in \widetilde{H}^{\gamma}(D) \cap L^{\infty}(D), 0 \leq \gamma \leq 1, \gamma \neq 1 / 2$. Then there is an $h_{0}>0$ such that for all $h \leq h_{0}$, the solution $u_{h}^{r}$ to problem (4.1) satisfies for any $\beta \in[1-\alpha / 2,1 / 2)$, with $\ell(\beta, \gamma)=: \min (\alpha-1+\beta, \gamma)$

$$
\left\|u^{r}-u_{h}^{r}\right\|_{L^{2}(D)}+h^{\alpha / 2-1+\beta}\left\|u^{r}-u_{h}^{r}\right\|_{\widetilde{H}^{\alpha / 2}(D)} \leq C h^{\min (2, \alpha+\ell(\beta, \gamma))-1+\beta}\|f\|_{\widetilde{H}^{\gamma}(D)} .
$$

Proof. The error estimate in the $\widetilde{H}^{\alpha / 2}(D)$-norm follows directly from Céa's lemma, (4.8) and the Galerkin orthogonality. Specifically, for all $h \leq h_{0}$ and any $\chi \in V_{h}$ we have by Galerkin orthogonality, (3.9) and (4.8)

$$
\begin{aligned}
\frac{\delta}{2}\left\|u_{h}^{r}-\chi\right\|_{V} & \leq C \sup _{v \in V_{h}} \frac{a_{r}\left(u_{h}^{r}-\chi, v\right)}{\|v\|_{V}} \\
& \leq C \sup _{v \in V_{h}} \frac{a_{r}\left(u^{r}-\chi, v\right)}{\|v\|_{V}} \leq C\left\|u^{r}-\chi\right\|_{V} .
\end{aligned}
$$

Hence the triangle inequality yields for all $\chi \in V_{h}$

$$
\left\|u^{r}-u_{h}^{r}\right\|_{V} \leq\left\|u^{r}-\chi\right\|_{V}+\left\|\chi-u_{h}^{r}\right\|_{V} \leq C\left\|u^{r}-\chi\right\|_{V} .
$$

Then the desired $\widetilde{H}^{\alpha / 2}(D)$-estimate follows from Lemma 2.4 by

$$
\begin{aligned}
\left\|u^{r}-u_{h}^{r}\right\|_{V} & \leq C \inf _{\chi \in V_{h}}\left\|u^{r}-\chi\right\|_{V} \\
& \leq C h^{\min (2, \alpha+\ell(\beta, \gamma))-\alpha / 2}\left\|u^{r}\right\|_{H^{\alpha+\ell(\beta, \gamma)}} \\
& \leq C h^{\min (2, \alpha+\ell(\beta, \gamma))-\alpha / 2}\|f\|_{\tilde{H}^{\gamma}(D)}
\end{aligned}
$$


Then we apply Nitsche's trick to establish the $L^{2}(D)$-error estimate. To this end, we consider the adjoint problem (3.15) with $f=u^{r}-u_{h}^{r}$, i.e.,

$$
\left\|u^{r}-u_{h}^{r}\right\|_{L^{2}(D)}^{2}=a_{r}\left(u^{r}-u_{h}^{r}, w\right)=a_{r}\left(u^{r}-u_{h}^{r}, w-w_{h}\right),
$$

for any $w_{h} \in V_{h}$. Then Lemma 2.4, Theorem 3.6 and (3.9) yield for any $\beta \in[1-\alpha / 2,1 / 2)$

$$
\begin{aligned}
\left\|u^{r}-u_{h}^{r}\right\|_{L^{2}(D)}^{2} & \leq\left\|u^{r}-u_{h}^{r}\right\|_{V} \inf _{w_{h} \in V_{h}}\left\|w-w_{h}\right\|_{V} \\
& \leq C h^{\min (2, \alpha+\ell(\beta, \gamma))-1+\beta}\|f\|_{\widetilde{H}^{\gamma}(D)}\left\|u^{r}-u_{h}^{r}\right\|_{L^{2}(D)} .
\end{aligned}
$$

This completes the proof of the theorem.

Now we turn to the reconstruction $\mu_{h}$ of the singularity strength $\mu$.

Lemma 4.3. Let the assumptions in Theorem 4.2 hold. Then there is an $h_{0}>0$ such that for all $h \leq h_{0}$, the solution $\mu_{h}$ satisfies that for any $\beta \in[1-\alpha / 2,1 / 2)$, with $\ell(\beta, \gamma)=: \min (\alpha-1+\beta, \gamma)$

$$
\left|\mu-\mu_{h}\right| \leq C h^{\min (2, \alpha+\ell(\beta, \gamma))-1+\beta}\|f\|_{\widetilde{H}^{\gamma}(D)} .
$$

Proof. We first recall that $q\left(u^{r}-u_{h}^{r}\right) \in L^{2}(D)$ since $q \in L^{\infty}(D)$ and $u^{r}-u_{h}^{r} \in L^{2}(D)$. Thus by Sobolev imbedding theorem, we have

$$
\left|\mu-\mu_{h}\right|=\left.\left|c_{0}\right|\right|_{0} I_{x}^{\alpha}\left(q\left(u^{r}-u_{h}^{r}\right)\right)(1) \mid \leq C\left\|_{0} I_{x}^{\alpha}\left(q\left(u^{r}-u_{h}^{r}\right)\right)\right\|_{\widetilde{H}_{L}^{\alpha}(D)} .
$$

Then by Theorems 2.1(c) and 4.2, we have for any $\beta \in[1-\alpha / 2,1 / 2)$

$$
\left|\mu-\mu_{h}\right| \leq C\left\|u^{r}-u_{h}^{r}\right\|_{L^{2}(D)} \leq C h^{\min (2, \alpha+\ell(\beta, \gamma))-1+\beta}\|f\|_{\widetilde{H}^{\gamma}(D)} .
$$

Now we can derive a first error estimate for the approximation $u_{h}$ defined in (4.3).

Theorem 4.4. Let the assumptions in Theorem 4.2 hold. Then there is an $h_{0}>0$ such that for all $h \leq h_{0}$, the solution $u_{h}$ satisfies that for any $\beta \in[1-\alpha / 2,1 / 2)$, with $\ell(\beta, \gamma)=: \min (\alpha-1+\beta, \gamma)$

$$
\left\|u-u_{h}\right\|_{L^{2}(D)}+h^{\alpha / 2-1+\beta}\left\|u-u_{h}\right\|_{\widetilde{H}^{\alpha / 2}(D)} \leq C h^{\min (2, \alpha+\ell(\beta, \gamma))-1+\beta}\|f\|_{\tilde{H}^{\gamma}(D)} .
$$

Proof. The definitions of $u$ and $u_{h}$ imply

$$
\left\|u-u_{h}\right\|_{\widetilde{H}^{\alpha / 2}(D)} \leq\left\|u^{r}-u_{h}^{r}\right\|_{\widetilde{H}^{\alpha / 2}(D)}+\left|\mu-\mu_{h}\right|\left\|x^{\alpha-1}-x^{2}\right\|_{\widetilde{H}^{\alpha / 2}(D)} .
$$

Then the desired estimate follows from Theorem 4.2 and Lemma 4.3. The proof of the $L^{2}(D)$-estimate is analogously and hence omitted.

Remark 4.5. By Theorem 4.4, for $q \in L^{\infty}(D)$ and $f \in L^{2}(D)$, the $L^{2}(D)$ - and $\widetilde{H}^{\alpha / 2}(D)$-norm of the error can be respectively almost of order $O\left(h^{\alpha-1 / 2}\right)$ and $O\left(h^{\alpha / 2}\right)$, even though the solution $u$ is only in $\widetilde{H}_{L}^{\alpha-1+\beta}(D)$ for $\beta \in[1-\alpha / 2,1 / 2)$, which is better than that for the standard Galerkin method by $O\left(h^{\alpha / 2}\right)$ for both the $L^{2}(D)$ and $\widetilde{H}^{\alpha / 2}(D)$ error estimates ( $c f$. Thm. 2.5). Hence, in comparison with the standard Galerkin FEM, the new approach does yield a higher-order convergence rate. 
Remark 4.6. In contrast to the standard Galerkin FEM, the convergence rate of the singularity reconstruction technique can be further picked up, if $q$ and $f$ are smoother. Specifically, for sufficiently smooth $q$ and $f$, the $L^{2}(D)$ and $\widetilde{H}^{\alpha / 2}(D)$-estimates are respectively almost $O\left(h^{\min (3 / 2,2 \alpha-1)}\right)$ and $O\left(h^{\min (2-\alpha / 2,3 \alpha / 2-1 / 2)}\right)$, even though the regularity of the solution $u$ remains only in $\widetilde{H}_{L}^{\alpha-1+\beta}(D) \cap \widetilde{H}^{\alpha / 2}(D)$. The $L^{2}(D)$ estimate is not sharp, due to the low global regularity of the adjoint problem. However, we note that the adjoint problem has its leading singularity concentrated only at one point, i.e., $x=1$. This structure is not exploited in Nitsche's argument. It is still unclear how to incorporate the structure. One possibility is first to develop local error estimates.

Last we shall derive an $L^{\infty}(D)$-error estimate for the approximation $u_{h}$. To this end, we consider Green's function for problem (3.16), i.e., to find $G(x, y)$ such that for any fixed $x \in D$

$$
-{ }_{y}^{R} D_{1}^{\alpha} G(x, y)+q(y) G(x, y)+\frac{(1-y)^{\alpha-1}}{\Gamma(\alpha)}(Q, G(x, \cdot))=\delta_{x}(y), \quad y \in D,
$$

with the boundary condition $G(x, 0)=G(x, 1)=0$. We note that the variational formulation of Green's function is given by: find $G(x, y) \in V \equiv \widetilde{H}^{\alpha / 2}(D)$ such that for all $x \in D$

$$
a_{r}(v, G(x, \cdot))=\left\langle v, \delta_{x}\right\rangle=v(x) \quad \forall v \in V .
$$

The existence and uniqueness of Green's function $G(x, y) \in V$ follows directly from Theorem 3.6 and the fact that $\delta_{x} \in V^{*}$. Further, when $q \equiv 0$ the problem reduces to the standard fractional boundary value problem, for which the Green's function has the explicit representation $(2.13)$ and $G(x, \cdot) \in \widetilde{H}_{R}^{\alpha-1+\beta}(D)$ with $\beta \in[1-\alpha / 2,1 / 2)$. In case of a general $q \neq 0$, the Green's function $G(x, y)$ to problem (4.11) satisfies

$$
-{ }_{y}^{R} D_{1}^{\alpha} \alpha G(x, y)=\delta_{x}(y)-q(y) G(x, y)-\frac{(1-y)^{\alpha-1} q(y)}{\Gamma(\alpha)}(Q, G(x, \cdot)) .
$$

Hence, for $q \in L^{\infty}(D), q(\cdot) G(x, \cdot) \in L^{2}(D)$ and ${ }_{y} I_{1}^{\alpha} \delta_{x} \in \widetilde{H}_{R}^{\alpha-1+\beta}(D)$, and thus Green's function $G(x, \cdot)$ belongs to $\widetilde{H}_{R}^{\alpha-1+\beta}(D)$ with $\beta \in[1-\alpha / 2,1 / 2)$.

Now we can state an error estimate in the $L^{\infty}(D)$-norm for the approximation $u_{h}$.

Theorem 4.7. Let the assumptions in Theorem 4.2 hold. Then there is an $h_{0}>0$ such that for all $h \leq h_{0}$, the solution $u_{h}$ satisfies that for any $\beta \in[1-\alpha / 2,1 / 2)$, with $\ell(\beta, \gamma)=: \min (\alpha-1+\beta, \gamma)$, there holds

$$
\left\|u-u_{h}\right\|_{L^{\infty}(D)} \leq C h^{\min (2, \alpha+\ell(\beta, \gamma))-1+\beta}\|f\|_{\widetilde{H}^{\gamma}(D)} .
$$

Proof. Like before, we first derive an $L^{\infty}(D)$-estimate of $u^{r}-u_{h}^{r}$. By the weak formulation of Green's function (4.12) and Galerkin orthogonality, we have for all $x \in D$ and $w_{h} \in V_{h}$

$$
\left(u^{r}-u_{h}^{r}\right)(x)=a_{r}\left(u^{r}-u_{h}^{r}, G(x, \cdot)\right)=a_{r}\left(u^{r}-u_{h}^{r}, G(x, \cdot)-w_{h}\right) .
$$

Then by (2.7), Lemma 2.4 and Theorem 3.4 we obtain for any $\beta \in[1-\alpha / 2,1 / 2)$

$$
\begin{aligned}
\left|\left(u^{r}-u_{h}^{r}\right)(x)\right| & \leq\left\|u^{r}-u_{h}^{r}\right\|_{V} \inf _{w_{h} \in V_{h}}\left\|G(x, \cdot)-w_{h}\right\|_{V} \\
& \leq C h^{\min (2, \alpha+\ell(\beta, \gamma))-1+\beta}\left\|u^{r}\right\|_{H^{\alpha+\ell(\beta, \gamma)}(D)} \\
& \leq C h^{\min (2, \alpha+\ell(\beta, \gamma))-1+\beta}\|f\|_{\tilde{H}^{\gamma}(D)}
\end{aligned}
$$

Then Lemma 4.3 yields

$$
\begin{aligned}
\left\|u-u_{h}\right\|_{L^{\infty}(D)} & \leq\left\|u^{r}-u_{h}^{r}\right\|_{L^{\infty}(D)}+\left|\mu-\mu_{h}\right|\left\|x^{\alpha-1}-x^{2}\right\|_{L^{\infty}(D)} \\
& \leq C h^{\min (2, \alpha+\ell(\beta, \gamma))-1+\beta}\|f\|_{\widetilde{H}^{\gamma}(D)} .
\end{aligned}
$$

This completes the proof of the theorem. 
Remark 4.8. In view of Theorems 2.7 and 4.7, for $f \in L^{2}(D)$, the $L^{\infty}(D)$-norm of the error is one half order higher for the new strategy than the standard Galerkin FEM. If $f$ is smoother (in the space $\widetilde{H}_{L}^{s}(D)$ ), the new technique yields an even higher convergence rate due to the enhance regularity of the regular part $u^{r}$. Further, numerically, a superconvergence phenomenon in the $L^{\infty}(D)$-norm is observed for the case $\alpha+\ell(\beta, \gamma)>2$.

\section{EXTENSION TO MIXED BOUNDARY VALUE PROBLEM}

The reconstruction technique is very versatile, and it can be straightforwardly extended to other type boundary conditions. We illustrate it with the following boundary value problem with a mixed boundary condition: find $u \in L^{2}(D)$ such that

$$
\begin{aligned}
-{ }_{0}^{R} D_{x}^{\alpha} u+q u & =f \quad \text { in } D \\
{ }_{0}^{R} D_{x}^{\alpha-1} u(0)=u(1) & =0,
\end{aligned}
$$

with $\alpha \in(3 / 2,2)$. The choice $\alpha \in(3 / 2,2)$ is to ensure that problem (5.1) has a solution $u$ in $L^{2}(D)$; see the discussions below. To the best of our knowledge, the case of a mixed boundary condition like problem (5.1) has not been analyzed in the literature.

\subsection{Well-posedness}

We first discuss the well-posedness of problem (5.1) and the regularity pickup. Let $g={ }_{0} I_{x}^{\alpha} f$. By (Sect. 3 of [11]) (also [15], p. 44, Thm. 2.4), we have ${ }_{0}^{R} D_{x}^{\alpha} g=f$. This together with the identities $\left({ }_{0}^{R} D_{x}^{\alpha-1} g\right)(0)=0$ and ${ }_{0}^{R} D_{x}^{\alpha-1} x^{\alpha-2}=0$ implies that in case of $q \equiv 0$, problem (5.1) has a solution

$$
u(x)=-\left({ }_{0} I_{x}^{\alpha} f\right)(x)+\left({ }_{0} I_{x}^{\alpha} f\right)(1) x^{\alpha-2},
$$

which belongs to $\widetilde{H}_{L}^{\alpha-3 / 2-\epsilon}(D) \subset L^{2}(D), \epsilon \in(0, \alpha-3 / 2)$. To analyze the well-posedness of the problem, we introduce the following function spaces

$$
U=\left(\widetilde{H}_{L}^{\alpha-1}(D) \oplus\left\{x^{\alpha-2}\right\}\right) \cap\{u(1)=0\} \quad \text { and } \quad V=\widetilde{H}_{R}^{1}(D) .
$$

Clearly, any element $u \in U$ can be uniquely represented by $u=u_{0}-u_{0}(1) x^{\alpha-2}$ for some $u_{0} \in \widetilde{H}_{L}^{\alpha-1}(D)$. The next lemma shows that the functional

$$
\|u\|_{U}=\left\|_{0}^{R} D_{x}^{\alpha-1} u_{0}\right\|_{L^{2}(D)}
$$

defines a norm in the space $U$.

Lemma 5.1. $U$ is a Banach space with the norm $\|\cdot\|_{U}$.

Proof. The triangle inequality and absolute homogeneity follow immediately. It suffices to show that $\|u\|_{U}=0$ implies $u=0$ for all $u \in U$. By Theorem 2.1(b), we have

$$
\left\|_{0}^{R} D_{x}^{\alpha-1} \varphi\right\|_{L^{2}(D)} \leq C\|\varphi\|_{\widetilde{H}_{L}^{\alpha-1}(D)} \quad \forall \varphi \in \widetilde{H}_{L}^{\alpha-1}(D) .
$$

Meanwhile, for $\varphi \in \widetilde{H}_{L}^{\alpha-1}(D)$, by setting $v={ }_{0}^{R} D_{x}^{\alpha-1} \varphi$, we have $\varphi={ }_{0} I_{x}^{\alpha-1} v(x)$. By Theorem 2.1(c), there holds

$$
\|\varphi\|_{\widetilde{H}_{L}^{\alpha-1}(D)}=\left\|I_{0}^{\alpha-1} v\right\|_{\widetilde{H}_{L}^{\alpha-1}(D)} \leq C\|v\|_{L^{2}(D)}=C\left\|_{0}^{R} D_{x}^{\alpha-1} \varphi\right\|_{L^{2}(D)} .
$$

Thus the seminorm $\left\|_{0}^{R} D_{x}^{\alpha-1} \cdot\right\|_{L^{2}(D)}$ is equivalent to the norm $\|\cdot\|_{\tilde{H}_{L}^{\alpha-1}(D)}$ in the space $\widetilde{H}_{L}^{\alpha-1}(D)$. Hence,

$$
\|u\|_{U}=\left\|_{0}^{R} D_{x}^{\alpha-1} u_{0}\right\|_{L^{2}(D)}=0 \Rightarrow\left\|u_{0}\right\|_{\tilde{H}_{L}^{\alpha-1}(D)}=0 \Rightarrow u_{0}=0 \Rightarrow u=0 .
$$

Further, the completeness follows from the completeness of $\widetilde{H}_{L}^{\alpha-1}(D)$ and Sobolev imbedding theorem. 
Next we establish a stable variational formulation and derive the regularity pickup. We define a bilinear form $A(\cdot, \cdot): U \times V \rightarrow \mathbb{R}$ by

$$
A(u, v)=\left({ }_{0}^{R} D_{x}^{\alpha-1} u, \quad v^{\prime}\right) .
$$

It can be verified directly that the representation (5.2) satisfies the following Petrov-Galerkin's formulation

$$
A(u, v)=(f, v) \quad \forall v \in V .
$$

Now we show the inf-sup condition of the bilinear form $A(\cdot, \cdot)$. For any fixed $u \in U$, by choosing $v_{u}={ }_{0} I_{x}^{2-\alpha} u-$ $\left({ }_{0} I_{x}^{2-\alpha} u\right)(1) \in \widetilde{H}_{R}^{1}(D)$ we obtain

$$
\sup _{v \in V} \frac{A(u, v)}{\left\|v^{\prime}\right\|_{L^{2}(D)}} \geq \frac{A\left(u, v_{u}\right)}{\left\|v_{u}^{\prime}\right\|_{L^{2}(D)}}=\|u\|_{U} .
$$

Now for any nonzero $v \in V$, by choosing $u_{v}={ }_{0} I_{x}^{\alpha-1} v^{\prime}-\left({ }_{0} I_{x}^{\alpha-1} v^{\prime}\right)(1) x^{\alpha-2} \in U$ we have

$$
A\left(u_{v}, v\right)=\left\|v^{\prime}\right\|_{L^{2}(D)}>0,
$$

which implies that the inf-sup condition of the adjoint problem holds as well. Consequently, problem (5.5) with $q \equiv 0$ has a unique solution in the space $U$, and it can be represented by (5.2).

In the general case $q \neq 0$, we define

$$
a(u, v)=A(u, v)+(q u, v) .
$$

Then the variational formulation for problem (5.1) is given by: find $u \in U$ such that

$$
a(u, v)=(f, v) \quad \forall v \in V .
$$

To study the bilinear form $a(\cdot, \cdot)$, we make the following assumption analogous to Assumption 2.3.

Assumption 5.2. Let the bilinear form $a(u, v)$ with $u \in U$ and $v \in V$ satisfy

(a) The problem of finding $u \in U$ such that $a(u, v)=0$ for all $v \in V$ has only the trivial solution $u \equiv 0$.

$\left(\mathrm{a}^{*}\right)$ The problem of finding $v \in V$ such that $a(u, v)=0$ for all $u \in U$ has only the trivial solution $v \equiv 0$.

Under Assumption 5.2, we have the following theorem.

Theorem 5.3. Let $\alpha \in(3 / 2,2), q \in \widetilde{H}^{\gamma}(D), f \in \widetilde{H}_{L}^{\gamma}(D), 0 \leq \gamma \leq 1, \gamma \neq 1 / 2$, and Assumption 5.2 hold. Then there exists a unique solution $u=u_{0}-u_{0}(1) x^{\alpha-2} \in U$ with $u_{0} \in \widetilde{H}_{L}^{\alpha+\beta}(D)$ to problem (5.6) and further, for any $\beta \in[2-\alpha, 1 / 2)$, with $\ell_{n}(\beta, \gamma)=: \min (\alpha-2+\beta, \gamma)$, there holds

$$
\left\|u_{0}\right\|_{\widetilde{H}_{L}^{\alpha+\ell_{n}(\beta, \gamma)}(D)} \leq C\|f\|_{\widetilde{H}_{L}^{\gamma}(D)}
$$

Proof. Under Assumption 5.2, the proof of uniqueness and existence of a solution $u \in U$ is similar to that of Theorem 3.3, and hence omitted. By Lemma 2.2, the function $q u \in \widetilde{H}^{\min (\alpha-2+\beta, \gamma)}(D)$. Then the regularity of $u_{0}$ follows from the representation $u_{0}(x)=-\left({ }_{0} I_{x}^{\alpha}(f-q u)\right)(x)$ and Theorem 2.1(c).

\subsection{New variational formulation and FE approximation}

A direct application of the variational formulation (5.6) is inefficient for the numerical solution of problem (5.1), due to the low solution regularity, as a consequence of the presence of the term $x^{\alpha-2}$. To enhance the efficiency, we employ the singularity reconstruction technique, and consider the following splitting

$$
u=u^{r}+\mu\left(x^{\alpha-2}-x^{2}\right) .
$$


Repeating the arguments in Section 3 yields the following integro-differential problem for the regular part $u^{r}$

$$
\begin{gathered}
-{ }_{0}^{R} D_{x}^{\alpha} u^{r}+q u^{r}+\left({ }_{0} I_{x}^{\alpha}\left(q u^{r}\right)\right)(1) Q=\widetilde{f} \quad \text { in } D, \\
u^{r}(0)=u^{r}(1)=0 .
\end{gathered}
$$

It is worth noting that problem (5.8) has a homogeneous Dirichlet boundary condition like in Section 3. In problem (5.8), the functions $Q(x)$ and $\widetilde{f}(x)$ are given respectively by

$$
\begin{aligned}
& Q(x)=c_{0} c_{1}(x)-c_{0} q(x)\left(x^{\alpha-2}-x^{2}\right) \in L^{2}(D), \\
& \widetilde{f}(x)=f(x)+c_{0} c_{1}(x)\left({ }_{0} I_{x}^{\alpha} f\right)(1)-c_{0}\left({ }_{0} I_{x}^{\alpha} f\right)(1) q(x)\left(x^{\alpha-2}-x^{2}\right) \in L^{2}(D),
\end{aligned}
$$

where the constant $c_{0}$ and the function $c_{1}(x)$ are respectively defined by

$$
c_{0}=\frac{1}{1+{ }_{0} I_{x}^{\alpha}\left(q\left(x^{\alpha-2}-x^{2}\right)\right)(1)} \quad \text { and } \quad c_{1}={ }_{0}^{R} D_{x}^{\alpha}\left(x^{\alpha-2}-x^{2}\right)=-\frac{2}{\Gamma(3-\alpha)} x^{2-\alpha} .
$$

The preceding discussions indicate that the singularity reconstruction technique handles the mixed problem in the same manner as for the Dirichlet's problem, with their only difference lying in replacing the function $x^{\alpha-1}$ in the Dirichlet case with $x^{\alpha-2}$ in the mixed case. This shows clearly its versatility. Hence, the corresponding bilinear form $a_{r}(\cdot, \cdot)$ is given by

$$
a_{r}(u, v)=A(u, v)+(q u, v)+{ }_{0} I_{x}^{\alpha}(q u)(1)(Q, v) \quad \forall u, v \in V \equiv \widetilde{H}^{\alpha / 2}(D),
$$

where the bilinear form $a_{r}(\cdot, \cdot)$ is defined in (3.6), and the function $Q$ is defined in (5.9). Then the variational problem for the regular part $u^{r}$ reads: find $u^{r} \in V$ satisfying

$$
a_{r}\left(u^{r}, v\right)=(\tilde{f}, v) \quad \forall v \in V .
$$

Once the regular part $u^{r}$ is determined, the singularity strength $\mu$ can be obtained by

$$
\mu=c_{0}\left({ }_{0} I_{x}^{\alpha}\left(f-q u^{r}\right)\right)(1),
$$

Finally the solution $u$ of the source problem (5.1) is recovered by (5.7). The variational formulation (5.11) lends itself to the following discrete problem: find $u_{h}^{r} \in V_{h}$ such that

$$
a_{r}\left(u_{h}^{r}, v\right)=(\tilde{f}, v) \quad \forall v \in V_{h},
$$

where the bilinear form $a_{r}(\cdot, \cdot)$ and the source term $\tilde{f} \in L^{2}(D)$ are defined in (5.11) and (5.9), respectively. Then we construct a finite element approximation $\mu_{h}$ to the strength $\mu$ of the singular part $u^{s}$ by

$$
\mu_{h}=c_{0}\left({ }_{0} I_{x}^{\alpha}\left(f-q u_{h}^{r}\right)\right)(1)
$$

where the constant $c_{0}$ is defined in (5.10). Last, we construct an approximate solution $u_{h}$ to (5.1) by

$$
u_{h}=u_{h}^{r}+\mu_{h}\left(x^{\alpha-2}-x^{2}\right) .
$$

We have the following $L^{\infty}(D), L^{2}(D)$ and $\widetilde{H}^{\alpha / 2}(D)$-norm error estimates on the Galerkin approximation $u_{h}^{r}$ and the singularity strength $\mu_{h}$. The proof is omitted since it is identical to that in Section 4.

Theorem 5.4. Let Assumption 3.1 hold, $f \in \widetilde{H}^{\gamma}(D)$, and $q \in \widetilde{H}^{\gamma}(D) \cap L^{\infty}(D), 0 \leq \gamma \leq 1, \gamma \neq 1 / 2$. Then there is an $h_{0}>0$ such that for all $h \leq h_{0}$, the solution $u_{h}^{r}$ to problem (5.13) satisfies for any $\beta \in[1-\alpha / 2,1 / 2)$, with $\ell_{n}(\beta, \gamma)=: \min (\alpha-2+\beta, \gamma)$

$$
\left\|u^{r}-u_{h}^{r}\right\|_{L^{2}(D)}+h^{\alpha / 2-1+\beta}\left\|u^{r}-u_{h}^{r}\right\|_{\widetilde{H}^{\alpha / 2}(D)} \leq C h^{\min \left(2, \alpha+\ell_{n}(\beta, \gamma)\right)-1+\beta}\|f\|_{\widetilde{H}^{\gamma}(D)} .
$$

Further, let $\mu$ and $\mu_{h}$ be defined in (5.12) and (5.14). Then there holds

$$
\left|\mu-\mu_{h}\right| \leq C h^{\min \left(2, \alpha+\ell_{n}(\beta, \gamma)\right)-1+\beta}\|f\|_{\widetilde{H}^{\gamma}(D)} .
$$


Remark 5.5. The $L^{2}(D)$ convergence rate of the approximation $u_{h}$ defined in (5.15) follows directly from Theorem 5.4. Like the Dirichlet case, the $L^{2}(D)$ error estimate is suboptimal, due to the limited regularity of the adjoint solution. However, the $L^{\infty}(D)$ and $\widetilde{H}_{R}^{\alpha / 2}(D)$ error estimates do not follow, since the exact solution $u$ is generally neither bounded nor in $\widetilde{H}_{R}^{\alpha / 2}(D)$, due to the presence of the term $x^{\alpha-2}$ in the solution representation.

\section{Numerical RESUlts AND DisCussions}

In this section we present numerical experiments to verify our theoretical findings. We consider the following three different source terms:

(a) The source term $f(x)=x(1-x)$ belongs to the space $\widetilde{H}^{1+\epsilon}(D)$ for any $\epsilon \in[0,1 / 2)$.

(b) The source term $f(x)=\chi_{[0,1 / 2]}$ belongs to the space $\widetilde{H}^{\epsilon}(D)$ for any $\epsilon \in[0,1 / 2)$.

(c) The source term $f(x)=x^{-1 / 4}$ belongs to the space $\widetilde{H}^{\epsilon}(D)$ for any $\epsilon \in[0,1 / 4)$.

The computations were performed on uniform meshes of mesh sizes $h=1 / 2^{k}, k=5,6, \ldots, 10$. We note that if the potential $q$ is zero, the exact solution $u$ can be computed directly using the solution representation (2.3), and similarly the regular part $u^{r}$ can be evaluated in closed form. For the case $q \neq 0$, the exact solution is not available explicitly, and hence we compute the reference solution using a very refined mesh with a mesh size $h=1 / 2^{13}$. For each example, we consider three different $\alpha$ values, i.e., $5 / 4,3 / 2$ and $7 / 4$, and present the $L^{2}(D)$, $\widetilde{H}^{\alpha / 2}(D)$, and $L^{\infty}(D)$-norm of the error $e=u^{r}-u_{h}^{r}$ of the regular part and the error $\left|\mu-\mu_{h}\right|$ of the singularity strength $\mu$ separately.

\subsection{Numerical experiments for example (a)}

We begin with the simple case $q=0$. By the representations (2.3), the exact solution $u(x)$ is given by

$$
u(x)=\frac{1}{\Gamma(\alpha+2)}\left(x^{\alpha-1}-x^{\alpha+1}\right)-\frac{2}{\Gamma(\alpha+3)}\left(x^{\alpha-1}-x^{\alpha+2}\right),
$$

and it belongs to $\widetilde{H}_{L}^{\alpha-1+\beta}(D)$ with $\beta \in[1-\alpha / 2,1 / 2)$ due to the presence of the term $x^{\alpha-1}$. Thus the standard Galerkin FEM converges slowly; see ([11], Tab. 1) and Table 1. In the table, rate refers to the empirical convergence rate when the mesh size $h$ halves, and the numbers in the bracket denote theoretical rates. The results in Table 1 indicate that the $L^{\infty}(D)$ estimate in Theorem 2.7 is sharp. By the definition (3.1), in the singularity splitting, the regular part $u^{r}$ and the singular part $u^{s}$ are given respectively by

$$
u^{r}=\frac{2\left(x^{\alpha+2}-x^{2}\right)}{\Gamma(\alpha+3)}+\frac{x^{2}-x^{\alpha+1}}{\Gamma(\alpha+2)} \quad \text { and } \quad \mu u^{s}=\left(\frac{1}{\Gamma(\alpha+2)}-\frac{2}{\Gamma(\alpha+3)}\right)\left(x^{\alpha-1}-x^{2}\right) .
$$

In particular, the regular part $u^{r}$ belongs to $H^{2}(D)$. Since the singularity strength $\mu=1 / \Gamma(\alpha+2)-2 / \Gamma(\alpha+3)$ does not depend on the regular part $u^{r}$, it suffices to check errors for $e=u^{r}-u_{h}^{r}$. In Table 2 we show the errors $\|e\|_{L^{2}(D)},\|e\|_{\tilde{H}^{\alpha / 2}(D)}$ and $\|e\|_{L^{\infty}(D)}$. The numerical results show $O\left(h^{2}\right), O\left(h^{2-\alpha / 2}\right)$ and $O\left(h^{2}\right)$ for the $L^{2}(D)$, $\widetilde{H}^{\alpha / 2}(D)$ and $L^{\infty}(D)$-norms of the error, respectively. The $\widetilde{H}^{\alpha / 2}(D)$ estimate is fully confirmed; however, the $L^{2}(D)$ and $L^{\infty}(D)$ estimates are suboptimal: the empirical ones are one half order higher than the theoretical ones. The suboptimality is attributed to the low regularity of the adjoint solution, used in Nitsche's trick.

Next we check the problem with the potential $q=x(1-x) \in \widetilde{H}^{1+\epsilon}(D), \epsilon \in[0,1 / 2)$. Thus by Theorem 3.4, the regular part $u^{r}$ belongs to $H^{2}(D)$, and Theorems 4.4 and 4.7 predict almost $O\left(h^{3 / 2}\right), O\left(h^{2-\alpha / 2}\right)$ and $O\left(h^{3 / 2}\right)$ for the $L^{2}(D), \widetilde{H}^{\alpha / 2}(D)$, and $L^{\infty}(D)$-norms of the error, respectively. The numerical results fully confirm the $\widetilde{H}^{\alpha / 2}(D)$ estimate, but show one half order higher convergence for the $L^{2}(D)$ and $L^{\infty}(D)$-norm of the error, $c f$. Table 3. Further, we observe that the influence of the potential term on the approximation error is negligible. The numerical results in Table 4 show that the error $\left|\mu_{h}-\mu\right|$ of the reconstructed singular strength $\mu$ achieves a second-order convergence, which is higher than the theoretical rate from Lemma 4.3 by one half order. 
TABLE 1 . The $L^{\infty}(D)$-norm of the error by the standard Galerkin approximation for example (a) with $q=0, \alpha=1.25,1.5,1.75, h=1 / 2^{k}$.

\begin{tabular}{cccccccc}
\hline$k$ & 5 & 6 & 7 & 8 & 9 & 10 & rate \\
\hline$\alpha=1.25$ & $2.91 \mathrm{e}-2$ & $2.44 \mathrm{e}-2$ & $2.05 \mathrm{e}-2$ & $1.73 \mathrm{e}-2$ & $1.45 \mathrm{e}-2$ & $1.22 \mathrm{e}-2$ & $\approx 0.25(0.25)$ \\
$\alpha=1.5$ & $4.87 \mathrm{e}-3$ & $3.44 \mathrm{e}-3$ & $2.42 \mathrm{e}-3$ & $1.71 \mathrm{e}-3$ & $1.21 \mathrm{e}-3$ & $8.55 \mathrm{e}-4$ & $\approx 0.50(0.50)$ \\
$\alpha=1.75$ & $7.46 \mathrm{e}-4$ & $4.37 \mathrm{e}-4$ & $2.59 \mathrm{e}-4$ & $1.54 \mathrm{e}-4$ & $9.16 \mathrm{e}-5$ & $5.44 \mathrm{e}-5$ & $\approx 0.75(0.75)$ \\
\hline
\end{tabular}

TABle 2. The $L^{2}(D)-, \tilde{H}^{\alpha / 2}(D)$ - and $L^{\infty}(D)$-norm of the error $e=u^{r}-u_{h}^{r}$ for example (a) with $q=0, \alpha=1.25,1.5,1.75, h=1 / 2^{k}$.

\begin{tabular}{ccccccccc}
\hline$\alpha$ & $k$ & 5 & 6 & 7 & 8 & 9 & 10 & rate \\
\hline 1.25 & $L^{2}$ & $6.56 \mathrm{e}-5$ & $1.64 \mathrm{e}-5$ & $4.11 \mathrm{e}-6$ & $1.03 \mathrm{e}-6$ & $2.56 \mathrm{e}-7$ & $6.33 \mathrm{e}-8$ & $\approx 2.00(1.50)$ \\
& $\tilde{H}^{\alpha / 2}$ & $2.98 \mathrm{e}-4$ & $1.11 \mathrm{e}-4$ & $4.23 \mathrm{e}-5$ & $1.62 \mathrm{e}-5$ & $6.21 \mathrm{e}-6$ & $2.39 \mathrm{e}-6$ & $\approx 1.36(1.38)$ \\
& $L^{\infty}$ & $1.16 \mathrm{e}-4$ & $2.92 \mathrm{e}-5$ & $7.33 \mathrm{e}-6$ & $1.84 \mathrm{e}-6$ & $4.59 \mathrm{e}-7$ & $1.15 \mathrm{e}-7$ & $\approx 2.00(1.50)$ \\
\hline 1.5 & $L^{2}$ & $3.62 \mathrm{e}-5$ & $9.16 \mathrm{e}-6$ & $2.31 \mathrm{e}-6$ & $5.79 \mathrm{e}-7$ & $1.45 \mathrm{e}-7$ & $3.59 \mathrm{e}-8$ & $\approx 2.00(1.50)$ \\
& $\tilde{H}^{\alpha / 2}$ & $4.58 \mathrm{e}-4$ & $1.90 \mathrm{e}-4$ & $7.92 \mathrm{e}-5$ & $3.32 \mathrm{e}-5$ & $1.39 \mathrm{e}-5$ & $5.28 \mathrm{e}-6$ & $\approx 1.25(1.25)$ \\
& $L^{\infty}$ & $7.58 \mathrm{e}-5$ & $1.92 \mathrm{e}-5$ & $4.81 \mathrm{e}-6$ & $1.21 \mathrm{e}-6$ & $3.02 \mathrm{e}-7$ & $7.55 \mathrm{e}-8$ & $\approx 1.99(1.50)$ \\
\hline \multirow{2}{*}{1.75} & $L^{2}$ & $1.59 \mathrm{e}-5$ & $4.11 \mathrm{e}-6$ & $1.05 \mathrm{e}-6$ & $2.69 \mathrm{e}-7$ & $6.84 \mathrm{e}-8$ & $1.72 \mathrm{e}-8$ & $\approx 1.97(1.50)$ \\
& $\tilde{H}^{\alpha / 2}$ & $5.57 \mathrm{e}-4$ & $2.54 \mathrm{e}-4$ & $1.16 \mathrm{e}-4$ & $5.30 \mathrm{e}-5$ & $2.43 \mathrm{e}-5$ & $1.11 \mathrm{e}-5$ & $\approx 1.13(1.13)$ \\
& $L^{\infty}$ & $4.32 \mathrm{e}-5$ & $1.10 \mathrm{e}-5$ & $2.77 \mathrm{e}-6$ & $6.96 \mathrm{e}-7$ & $1.74 \mathrm{e}-7$ & $4.36 \mathrm{e}-8$ & $\approx 2.00(1.50)$ \\
\hline
\end{tabular}

TABle 3. The $L^{2}(D)-, \tilde{H}^{\alpha / 2}(D)$ - and $L^{\infty}(D)$-norm of the error $e=u^{r}-u_{h}^{r}$ for example (a) with $q=x(1-x), \alpha=1.25,1.5,1.75, h=1 / 2^{k}$.

\begin{tabular}{ccccccccc}
\hline$\alpha$ & $k$ & 5 & 6 & 7 & 8 & 9 & 10 & rate \\
\hline 1.25 & $L^{2}$ & $6.42 \mathrm{e}-5$ & $1.61 \mathrm{e}-5$ & $4.02 \mathrm{e}-6$ & $1.00 \mathrm{e}-6$ & $2.51 \mathrm{e}-7$ & $6.19 \mathrm{e}-8$ & $\approx 2.01(1.50)$ \\
& $\tilde{H}^{\alpha / 2}$ & $2.80 \mathrm{e}-4$ & $1.04 \mathrm{e}-4$ & $3.96 \mathrm{e}-5$ & $1.51 \mathrm{e}-5$ & $5.81 \mathrm{e}-6$ & $2.23 \mathrm{e}-6$ & $\approx 1.38(1.38)$ \\
& $L^{\infty}$ & $1.13 \mathrm{e}-4$ & $2.84 \mathrm{e}-5$ & $7.12 \mathrm{e}-6$ & $1.78 \mathrm{e}-6$ & $4.46 \mathrm{e}-7$ & $1.12 \mathrm{e}-7$ & $\approx 2.00(1.50)$ \\
\hline 1.5 & $L^{2}$ & $3.50 \mathrm{e}-5$ & $8.86 \mathrm{e}-6$ & $2.23 \mathrm{e}-6$ & $5.61 \mathrm{e}-7$ & $1.40 \mathrm{e}-7$ & $3.48 \mathrm{e}-8$ & $\approx 2.01(1.50)$ \\
& $\tilde{H}^{\alpha / 2}$ & $4.34 \mathrm{e}-4$ & $1.80 \mathrm{e}-4$ & $7.50 \mathrm{e}-5$ & $3.14 \mathrm{e}-5$ & $1.32 \mathrm{e}-5$ & $5.52 \mathrm{e}-6$ & $\approx 1.25(1.25)$ \\
& $L^{\infty}$ & $7.35 \mathrm{e}-5$ & $1.86 \mathrm{e}-5$ & $4.67 \mathrm{e}-6$ & $1.17 \mathrm{e}-6$ & $2.93 \mathrm{e}-7$ & $7.33 \mathrm{e}-8$ & $\approx 2.00(1.50)$ \\
\hline 1.75 & $L^{2}$ & $1.54 \mathrm{e}-5$ & $3.97 \mathrm{e}-6$ & $1.02 \mathrm{e}-6$ & $2.61 \mathrm{e}-7$ & $6.62 \mathrm{e}-8$ & $1.66 \mathrm{e}-8$ & $\approx 1.97(1.50)$ \\
& $\tilde{H}^{\alpha / 2}$ & $5.34 \mathrm{e}-4$ & $2.43 \mathrm{e}-4$ & $1.11 \mathrm{e}-4$ & $5.08 \mathrm{e}-5$ & $2.33 \mathrm{e}-5$ & $1.06-5$ & $\approx 1.13(1.13)$ \\
& $L^{\infty}$ & $4.20 \mathrm{e}-5$ & $1.07 \mathrm{e}-5$ & $2.70 \mathrm{e}-6$ & $6.78 \mathrm{e}-7$ & $1.70 \mathrm{e}-7$ & $4.25 \mathrm{e}-8$ & $\approx 2.00(1.50)$ \\
\hline
\end{tabular}

Apart from the reconstruction technique, there are alternative strategies for enhancing the computational efficiency of the standard Galerkin FEM. Mesh grading is one such possible choice, and clearly, it preserves the variational formulation. In Table 5, we present numerical results of the standard Galerkin FEM using graded meshes. Specifically, we consider the grid $x_{j}=(j h)^{\delta}$ with some $\delta \geq 1, j=0,1, \ldots, m$. The choice $\delta=1$ corresponds to a uniform mesh, whereas the choice $\delta>1$ makes the mesh graded near $x=0$ to compensate the singularity of the solution. It is observed from Table 5 that the optimal convergence rate is indeed achieved for a sufficiently large $\delta$. Further, its accuracy is comparable with the reconstruction approach (cf. Tab. 3). However, computationally, for a graded mesh, it is far more expensive to form the stiffness matrix, since it lacks nice structure, whereas for a uniform mesh, it is Toeplitz (plus rank-one perturbation). Further, in the mesh grading approach, the singularity is hard wired in the implementation and cannot automatically adapted to the case of vanishing singularity: if the solution is indeed smooth instead weakly singular, then the use of a graded 
TABLE 4. $\left|\mu-\mu_{h}\right|$ for example (a) with $q=x(1-x), \alpha=1.25,1.5,1.75, h=1 / 2^{k}$.

\begin{tabular}{cccccccc}
\hline$k$ & 5 & 6 & 7 & 8 & 9 & 10 & rate \\
\hline$\alpha=1.25$ & $8.62 \mathrm{e}-6$ & $2.16 \mathrm{e}-6$ & $5.40 \mathrm{e}-7$ & $1.35 \mathrm{e}-7$ & $3.33 \mathrm{e}-8$ & $7.93 \mathrm{e}-9$ & $\approx 2.02(1.50)$ \\
$\alpha=1.5$ & $3.70 \mathrm{e}-6$ & $9.43 \mathrm{e}-7$ & $2.39 \mathrm{e}-7$ & $6.01 \mathrm{e}-8$ & $1.49 \mathrm{e}-8$ & $3.57 \mathrm{e}-9$ & $\approx 2.01(1.50)$ \\
$\alpha=1.75$ & $9.49 \mathrm{e}-7$ & $2.60 \mathrm{e}-7$ & $6.96 \mathrm{e}-8$ & $1.83 \mathrm{e}-8$ & $4.72 \mathrm{e}-9$ & $1.16 \mathrm{e}-9$ & $\approx 1.96(1.50)$ \\
\hline
\end{tabular}

TABLE 5. The $L^{2}(D)-, \tilde{H}^{\alpha / 2}(D)$ - and $L^{\infty}(D)$-norm of the error $u-u_{h}$ for example (a) with $q=x(1-x), \alpha=1.25,1.75, h=1 / 2^{k}$ and $\delta=2,5$, using graded meshes.

\begin{tabular}{cccccccccc}
\hline$\alpha$ & $\delta$ & $k$ & 3 & 4 & 5 & 6 & 7 & 8 & rate \\
\hline \multirow{4}{*}{1.25} & & $L^{2}$ & $5.18 \mathrm{e}-3$ & $1.59 \mathrm{e}-3$ & $5.41 \mathrm{e}-4$ & $1.86 \mathrm{e}-4$ & $6.34 \mathrm{e}-5$ & $2.28 \mathrm{e}-5$ & $\approx 1.56$ \\
& 2 & $\tilde{H}^{\alpha / 2}$ & $5.67 \mathrm{e}-2$ & $4.59 \mathrm{e}-2$ & $3.70 \mathrm{e}-2$ & $2.89 \mathrm{e}-2$ & $2.05 \mathrm{e}-2$ & $1.30 \mathrm{e}-2$ & $\approx 0.42$ \\
& & $L^{\infty}$ & $2.46 \mathrm{e}-2$ & $1.64 \mathrm{e}-2$ & $1.15 \mathrm{e}-2$ & $8.10 \mathrm{e}-3$ & $5.73 \mathrm{e}-3$ & $4.05 \mathrm{e}-3$ & $\approx 0.52$ \\
\cline { 2 - 9 } & & $L^{2}$ & $6.63 \mathrm{e}-3$ & $3.04 \mathrm{e}-3$ & $9.08 \mathrm{e}-4$ & $2.40 \mathrm{e}-4$ & $6.13 \mathrm{e}-5$ & $1.57 \mathrm{e}-5$ & $\approx 1.97$ \\
& 5 & $\tilde{H}^{\alpha / 2}$ & $1.79 \mathrm{e}-2$ & $6.16 \mathrm{e}-3$ & $2.22 \mathrm{e}-3$ & $7.69 \mathrm{e}-4$ & $2.63 \mathrm{e}-4$ & $9.31 \mathrm{e}-5$ & $\approx 1.52$ \\
& & $L^{\infty}$ & $9.65 \mathrm{e}-3$ & $5.00 \mathrm{e}-3$ & $1.61 \mathrm{e}-3$ & $4.42 \mathrm{e}-3$ & $1.15 \mathrm{e}-4$ & $2.98 \mathrm{e}-5$ & $\approx 1.92$ \\
\hline \multirow{5}{*}{1.75} & & $L^{2}$ & $3.47 \mathrm{e}-4$ & $8.24 \mathrm{e}-5$ & $2.06 \mathrm{e}-5$ & $5.29 \mathrm{e}-6$ & $1.38 \mathrm{e}-6$ & $3.58 \mathrm{e}-7$ & $\approx 1.98$ \\
& & $\tilde{H}^{\alpha / 2}$ & $5.34 \mathrm{e}-4$ & $2.43 \mathrm{e}-4$ & $1.11 \mathrm{e}-4$ & $5.08 \mathrm{e}-5$ & $2.33 \mathrm{e}-5$ & $1.06-5$ & $\approx 1.02$ \\
& & $L^{\infty}$ & $4.20 \mathrm{e}-5$ & $1.07 \mathrm{e}-5$ & $2.70 \mathrm{e}-6$ & $6.78 \mathrm{e}-7$ & $1.70 \mathrm{e}-7$ & $4.25 \mathrm{e}-8$ & $\approx 1.70$ \\
\cline { 2 - 9 } & & $L^{2}$ & $2.19 \mathrm{e}-3$ & $3.73 \mathrm{e}-4$ & $9.19 \mathrm{e}-5$ & $2.46 \mathrm{e}-5$ & $6.69 \mathrm{e}-6$ & $1.80 \mathrm{e}-6$ & $\approx 2.05$ \\
& $\tilde{H}^{\alpha / 2}$ & $1.41 \mathrm{e}-2$ & $6.53 \mathrm{e}-3$ & $3.07 \mathrm{e}-3$ & $1.41 \mathrm{e}-3$ & $6.47 \mathrm{e}-4$ & $2.96 \mathrm{e}-4$ & $\approx 1.11$ \\
& $L^{\infty}$ & $3.90 \mathrm{e}-3$ & $9.29 \mathrm{e}-4$ & $1.88 \mathrm{e}-4$ & $7.25 \mathrm{e}-5$ & $2.19 \mathrm{e}-5$ & $5.93 \mathrm{e}-6$ & $\approx 1.87$ \\
\hline
\end{tabular}

mesh is wasteful; whereas the reconstruction technique can adapt itself automatically to the case of a vanishing singularity by recovering $\mu=0$, which makes it more flexible than the one based on graded meshes.

\subsection{Numerical experiments for example (b)}

In Tables 6 and 7 we present numerical results for problem (b) with $q=x(1-x)$. Since the source term $f$ is in $\widetilde{H}^{\epsilon}(D), \epsilon \in[0,1 / 2)$, regular part $u^{r}$ is in $H^{2}(D)$ for $\alpha \in(3 / 2,2)$, whereas for $\alpha \in(1,3 / 2]$, $u^{r}$ is in the space $H^{\alpha+\epsilon}$ with $\epsilon \in[0,1 / 2)$. In Table 6 , the numerical results exhibit a convergence rate of the order $O\left(h^{2}\right)$ in the $L^{2}(D)$-norm, and $O\left(h^{2-\alpha / 2}\right)$ in the $\widetilde{H}^{\alpha / 2}(D)$-norm, for both $\alpha=1.5$ and 1.75 . Further, we observe that the $L^{2}(D)$ and $\widetilde{H}^{\alpha / 2}(D)$-errors are respectively only of order $O\left(h^{1.75}\right)$ and $O\left(h^{1.13}\right)$ for $\alpha=1.25$, due to a lack of regularity of the regular part $u^{r}$. The empirical $L^{2}(D)$ rate is one half order higher than the theoretical prediction. Further, for the case of $\alpha=1.75$, we observe a superconvergence phenomenon for the $L^{\infty}(D)$-estimate, whereas for the case $\alpha=1.5$ and 1.25 , the $L^{\infty}(D)$-estimate agrees with our convergence theory. These experiments indicate an $L^{\infty}(D)$-norm of the error in the form $O\left(h^{\min (2, \alpha+\ell(\beta, \gamma)-1 / 2)}\right)$, instead of $O\left(h^{\min (2, \alpha+\ell(\beta, \gamma))-1+\beta}\right)$ from Theorem 4.7, and a superconvergence phenomenon occurs if $\alpha+\ell(\beta, \gamma)>2$. Surprisingly, the error $\left|\mu-\mu_{h}\right|$ of the recovered singularity strength $\mu_{h}$ achieves a second-order convergence even for $\alpha=1.25$ ( $c f$. Tab. 7), which remains to be justified.

\subsection{Numerical experiments for example (c)}

In Tables 8 and 9 , we present numerical results for example (c) with $q=x(1-x)$. Since the source term $f(x)=x^{-1 / 4}$ is in $\widetilde{H}^{\epsilon}(D)$ with $\epsilon \in[0,1 / 4)$, by Theorem 3.4, the regular part $u^{r}$ is in $H^{\alpha+\epsilon}(D)$, which implies a convergence rate $O\left(h^{\alpha+\epsilon-1 / 2}\right), O\left(h^{\alpha / 2+\epsilon}\right)$ and $O\left(h^{\alpha+\epsilon-1 / 2}\right)$ of the $L^{2}(D)-, \widetilde{H}^{\alpha / 2}(D)$ - and $L^{\infty}(D)$-norm of the error, respectively (cf. Thms. 4.4 and 4.7). The $L^{2}(D), \widetilde{H}^{\alpha / 2}(D)$ and $L^{\infty}(D)$-errors achieve a rate $O\left(h^{\alpha+1 / 4}\right)$, $O\left(h^{\alpha / 2+1 / 4}\right)$ and $O\left(h^{\alpha-1 / 4}\right)$, respectively, for $\alpha=1.50$ and 1.75 . The convergence of the Galerkin approach slows down as the fractional order $\alpha$ tends to unity, due to the lower regularity pickup of the regular part $u^{r}$, but the 
TABle 6 . The $L^{2}(D)-, \tilde{H}^{\alpha / 2}(D)$ - and $L^{\infty}(D)$-norm of the error $e=u^{r}-u_{h}^{r}$ for example (b) with $q=x(1-x), \alpha=1.25,1.5,1.75, h=1 / 2^{k}$.

\begin{tabular}{ccccccccc}
\hline$\alpha$ & $k$ & 5 & 6 & 7 & 8 & 9 & 10 & rate \\
\hline 1.25 & $L^{2}$ & $1.45 \mathrm{e}-4$ & $4.17 \mathrm{e}-5$ & $1.21 \mathrm{e}-5$ & $3.52 \mathrm{e}-6$ & $1.03 \mathrm{e}-6$ & $3.03 \mathrm{e}-7$ & $\approx 1.77(1.25)$ \\
& $\tilde{H}^{\alpha / 2}$ & $1.51 \mathrm{e}-3$ & $7.11 \mathrm{e}-4$ & $3.33 \mathrm{e}-4$ & $1.55 \mathrm{e}-4$ & $7.18 \mathrm{e}-5$ & $3.33 \mathrm{e}-5$ & $\approx 1.11(1.13)$ \\
& $L^{\infty}$ & $8.59 \mathrm{e}-4$ & $3.89 \mathrm{e}-4$ & $1.71 \mathrm{e}-4$ & $7.36 \mathrm{e}-5$ & $3.14 \mathrm{e}-5$ & $1.33 \mathrm{e}-5$ & $\approx 1.23(1.25)$ \\
\hline 1.5 & $L^{2}$ & $7.18 \mathrm{e}-5$ & $1.87 \mathrm{e}-5$ & $4.83 \mathrm{e}-6$ & $1.25 \mathrm{e}-6$ & $3.20 \mathrm{e}-7$ & $8.16 \mathrm{e}-8$ & $\approx 1.96(1.50)$ \\
& $\tilde{H}^{\alpha / 2}$ & $1.93 \mathrm{e}-3$ & $8.85 \mathrm{e}-4$ & $4.02 \mathrm{e}-4$ & $1.81 \mathrm{e}-4$ & $8.06 \mathrm{e}-5$ & $3.57 \mathrm{e}-5$ & $\approx 1.17(1.25)$ \\
& $L^{\infty}$ & $3.55 \mathrm{e}-4$ & $1.38 \mathrm{e}-4$ & $5.21 \mathrm{e}-5$ & $1.92 \mathrm{e}-5$ & $7.01 \mathrm{e}-6$ & $2.52 \mathrm{e}-6$ & $\approx 1.47(1.50)$ \\
\hline \multirow{2}{*}{1.75} & $L^{2}$ & $2.78 \mathrm{e}-5$ & $6.93 \mathrm{e}-6$ & $1.72 \mathrm{e}-6$ & $4.24 \mathrm{e}-7$ & $1.04 \mathrm{e}-7$ & $2.55 \mathrm{e}-8$ & $\approx 2.02(1.50)$ \\
& $\tilde{H}^{\alpha / 2}$ & $1.57 \mathrm{e}-3$ & $7.41 \mathrm{e}-4$ & $3.47 \mathrm{e}-4$ & $1.62 \mathrm{e}-4$ & $7.48 \mathrm{e}-5$ & $3.44 \mathrm{e}-5$ & $\approx 1.11(1.13)$ \\
& $L^{\infty}$ & $1.19 \mathrm{e}-4$ & $4.11 \mathrm{e}-5$ & $1.36 \mathrm{e}-5$ & $4.39 \mathrm{e}-6$ & $1.39 \mathrm{e}-6$ & $4.36 \mathrm{e}-7$ & $\approx 1.68(1.50)$ \\
\hline
\end{tabular}

TABLE 7. $\left|\mu-\mu_{h}\right|$ for example (b) with $q=x(1-x), \alpha=1.25,1.5,1.75, h=1 / 2^{k}$.

\begin{tabular}{cccccccc}
\hline$k$ & 5 & 6 & 7 & 8 & 9 & 10 & rate \\
\hline$\alpha=1.25$ & $1.25 \mathrm{e}-5$ & $3.11 \mathrm{e}-6$ & $7.73 \mathrm{e}-7$ & $1.92 \mathrm{e}-7$ & $4.75 \mathrm{e}-8$ & $1.13 \mathrm{e}-8$ & $\approx 2.02(1.25)$ \\
$\alpha=1.5$ & $5.76 \mathrm{e}-6$ & $1.41 \mathrm{e}-6$ & $3.48 \mathrm{e}-7$ & $8.60 \mathrm{e}-8$ & $2.11 \mathrm{e}-8$ & $5.00 \mathrm{e}-9$ & $\approx 2.04(1.50)$ \\
$\alpha=1.75$ & $1.85 \mathrm{e}-6$ & $4.52 \mathrm{e}-7$ & $1.11 \mathrm{e}-7$ & $2.72 \mathrm{e}-8$ & $6.63 \mathrm{e}-9$ & $1.56 \mathrm{e}-9$ & $\approx 2.04(1.50)$ \\
\hline
\end{tabular}

TABLE 8. The $L^{2}(D)-, \tilde{H}^{\alpha / 2}(D)$ - and $L^{\infty}(D)$-norms of the error $e=u^{r}-u_{h}^{r}$ for example (c) with $q=x(1-x), \alpha=1.25,1.5,1.75, h=1 / 2^{k}$.

\begin{tabular}{ccccccccc}
\hline$\alpha$ & $k$ & 5 & 6 & 7 & 8 & 9 & 10 & rate \\
\hline 1.25 & $L^{2}$ & $1.76 \mathrm{e}-4$ & $4.39 \mathrm{e}-5$ & $1.10 \mathrm{e}-5$ & $2.74 \mathrm{e}-6$ & $6.82 \mathrm{e}-7$ & $1.69 \mathrm{e}-7$ & $\approx 2.00(1.00)$ \\
& $\tilde{H}^{\alpha / 2}$ & $1.61 \mathrm{e}-3$ & $6.16 \mathrm{e}-4$ & $2.36 \mathrm{e}-4$ & $9.09 \mathrm{e}-5$ & $3.50 \mathrm{e}-5$ & $1.35 \mathrm{e}-5$ & $\approx 1.38(0.88)$ \\
& $L^{\infty}$ & $2.76 \mathrm{e}-4$ & $6.92 \mathrm{e}-5$ & $1.73 \mathrm{e}-5$ & $4.33 \mathrm{e}-6$ & $1.08 \mathrm{e}-6$ & $2.71 \mathrm{e}-7$ & $\approx 2.00(1.00)$ \\
\hline 1.5 & $L^{2}$ & $1.53 \mathrm{e}-4$ & $4.32 \mathrm{e}-5$ & $1.23 \mathrm{e}-5$ & $3.58 \mathrm{e}-6$ & $1.04 \mathrm{e}-6$ & $3.05 \mathrm{e}-7$ & $\approx 1.76(1.25)$ \\
& $\tilde{H}^{\alpha / 2}$ & $4.18 \mathrm{e}-3$ & $2.11 \mathrm{e}-3$ & $1.07 \mathrm{e}-3$ & $5.40 \mathrm{e}-4$ & $2.73 \mathrm{e}-4$ & $1.38 \mathrm{e}-4$ & $\approx 0.98(1.00)$ \\
& $L^{\infty}$ & $7.25 \mathrm{e}-4$ & $3.33 \mathrm{e}-4$ & $1.48 \mathrm{e}-4$ & $6.41 \mathrm{e}-5$ & $2.74 \mathrm{e}-5$ & $1.16 \mathrm{e}-5$ & $\approx 1.21(1.25)$ \\
\hline 1.75 & $L^{2}$ & $8.91 \mathrm{e}-5$ & $2.33 \mathrm{e}-5$ & $6.05 \mathrm{e}-6$ & $1.56 \mathrm{e}-6$ & $4.02 \mathrm{e}-7$ & $1.02 \mathrm{e}-7$ & $\approx 1.95(1.50)$ \\
& $\tilde{H}^{\alpha / 2}$ & $5.16 \mathrm{e}-3$ & $2.59 \mathrm{e}-3$ & $1.28 \mathrm{e}-3$ & $6.31 \mathrm{e}-4$ & $3.08 \mathrm{e}-4$ & $1.49 \mathrm{e}-4$ & $\approx 1.05(1.13)$ \\
& $L^{\infty}$ & $4.47 \mathrm{e}-4$ & $1.76 \mathrm{e}-4$ & $6.67 \mathrm{e}-5$ & $2.47 \mathrm{e}-5$ & $9.03 \mathrm{e}-6$ & $3.29 \mathrm{e}-7$ & $\approx 1.45(1.50)$ \\
\hline
\end{tabular}

TABLE 9. $\left|\mu-\mu_{h}\right|$ for example (c) with $q=x(1-x), \alpha=1.25,1.5,1.75, h=1 / 2^{k}$.

\begin{tabular}{cccccccc}
\hline$k$ & 5 & 6 & 7 & 8 & 9 & 10 & rate \\
\hline$\alpha=1.25$ & $2.10 \mathrm{e}-5$ & $5.25 \mathrm{e}-6$ & $1.31 \mathrm{e}-6$ & $3.27 \mathrm{e}-7$ & $8.08 \mathrm{e}-8$ & $1.92 \mathrm{e}-8$ & $\approx 2.03(1.00)$ \\
$\alpha=1.5$ & $1.18 \mathrm{e}-5$ & $2.90 \mathrm{e}-6$ & $7.13 \mathrm{e}-7$ & $1.76 \mathrm{e}-7$ & $4.31 \mathrm{e}-8$ & $1.02 \mathrm{e}-8$ & $\approx 2.05(1.50)$ \\
$\alpha=1.75$ & $5.59 \mathrm{e}-6$ & $1.36 \mathrm{e}-6$ & $3.33 \mathrm{e}-7$ & $8.13 \mathrm{e}-8$ & $2.00 \mathrm{e}-8$ & $4.87 \mathrm{e}-9$ & $\approx 2.03(1.50)$ \\
\hline
\end{tabular}

empirical behavior still agrees well with the theoretical predictions. However, for $\alpha=1.25$, the approximation $u_{h}$ converges faster: a second-order convergence in both $L^{2}(D)$ and $L^{\infty}(D)$-norms, and an $O\left(h^{1.38}\right)$ rate in the $\widetilde{H}^{\alpha / 2}(D)$-norm. This is attributed to the fact that ${ }_{0} I_{x}^{5 / 4} x^{-1 / 4}=\Gamma(3 / 4) x$, which is actually much smoother than $\widetilde{H}_{L}^{5 / 4+\epsilon}(D)$, with $\epsilon \in[0,1 / 4)$, from Theorem 3.4. Interestingly, the approximation $\mu_{h}$ of the singularity strength $\mu$ is second order accurate ( $c f$. Tab. 9), despite the low regularity of the data. 
TABle 10. The $L^{2}(D)-, \tilde{H}^{\alpha / 2}(D)$ - and $L^{\infty}(D)$-norms of the error $e=u^{r}-u_{h}^{r}$ for example (c) with mixed boundary conditions, $q=x(1-x), \alpha=1.6,1.75,1.9, h=1 / 2^{k}$.

\begin{tabular}{ccccccccc}
\hline$\alpha$ & $k$ & 5 & 6 & 7 & 8 & 9 & 10 & rate \\
\hline 1.6 & $L^{2}$ & $1.14 \mathrm{e}-4$ & $3.19 \mathrm{e}-5$ & $8.89 \mathrm{e}-6$ & $2.47 \mathrm{e}-6$ & $6.86 \mathrm{e}-7$ & $1.90 \mathrm{e}-7$ & $\approx 1.85(1.35)$ \\
& $\tilde{H}^{\alpha / 2}$ & $4.51 \mathrm{e}-3$ & $2.31 \mathrm{e}-3$ & $1.17 \mathrm{e}-3$ & $5.85 \mathrm{e}-4$ & $2.91 \mathrm{e}-4$ & $1.44 \mathrm{e}-4$ & $\approx 0.98(0.90)$ \\
& $L^{\infty}$ & $6.06 \mathrm{e}-4$ & $2.64 \mathrm{e}-4$ & $1.10 \mathrm{e}-4$ & $4.48 \mathrm{e}-5$ & $1.78 \mathrm{e}-5$ & $7.19 \mathrm{e}-6$ & $\approx 1.31(1.35)$ \\
\hline 1.75 & $L^{2}$ & $8.24 \mathrm{e}-5$ & $2.17 \mathrm{e}-5$ & $5.68 \mathrm{e}-6$ & $1.47 \mathrm{e}-6$ & $3.80 \mathrm{e}-7$ & $9.73 \mathrm{e}-8$ & $\approx 1.95(1.50)$ \\
& $\tilde{H}^{\alpha / 2}$ & $4.95 \mathrm{e}-3$ & $2.50 \mathrm{e}-3$ & $1.25 \mathrm{e}-3$ & $6.17 \mathrm{e}-4$ & $3.02 \mathrm{e}-4$ & $1.46 \mathrm{e}-4$ & $\approx 1.03(1.13)$ \\
& $L^{\infty}$ & $4.51 \mathrm{e}-4$ & $1.78 \mathrm{e}-4$ & $6.72 \mathrm{e}-5$ & $2.49 \mathrm{e}-5$ & $9.07 \mathrm{e}-6$ & $3.30 \mathrm{e}-6$ & $\approx 1.45(1.50)$ \\
\hline 1.9 & $L^{2}$ & $5.50 \mathrm{e}-5$ & $1.42 \mathrm{e}-5$ & $3.61 \mathrm{e}-6$ & $9.10 \mathrm{e}-7$ & $2.28 \mathrm{e}-7$ & $5.63 \mathrm{e}-8$ & $\approx 1.99(1.50)$ \\
& $\tilde{H}^{\alpha / 2}$ & $4.68 \mathrm{e}-3$ & $2.39 \mathrm{e}-3$ & $1.20 \mathrm{e}-3$ & $6.00 \mathrm{e}-4$ & $2.97 \mathrm{e}-4$ & $1.46 \mathrm{e}-4$ & $\approx 1.00(1.05)$ \\
& $L^{\infty}$ & $2.82 \mathrm{e}-4$ & $1.02 \mathrm{e}-4$ & $3.54 \mathrm{e}-5$ & $1.20 \mathrm{e}-5$ & $4.02 \mathrm{e}-6$ & $1.31 \mathrm{e}-6$ & $\approx 1.56(1.50)$ \\
\hline
\end{tabular}

TABLE 11. $\left|\mu-\mu_{h}\right|$ for example (c) with mixed boundary conditions, $q=x(1-x), \alpha=$ $1.6,1.75,1.9, h=1 / 2^{k}$.

\begin{tabular}{cccccccc}
\hline$\alpha \backslash k$ & 5 & 6 & 7 & 8 & 9 & 10 & rate \\
\hline 1.6 & $3.69 \mathrm{e}-5$ & $3.40 \mathrm{e}-6$ & $4.00 \mathrm{e}-7$ & $9.82 \mathrm{e}-8$ & $2.41 \mathrm{e}-8$ & $5.88 \mathrm{e}-9$ & $\approx 2.03(1.00)$ \\
1.75 & $2.07 \mathrm{e}-5$ & $1.11 \mathrm{e}-6$ & $2.68 \mathrm{e}-7$ & $6.53 \mathrm{e}-8$ & $1.59 \mathrm{e}-8$ & $3.83 \mathrm{e}-9$ & $\approx 2.04(1.50)$ \\
1.9 & $5.59 \mathrm{e}-5$ & $1.36 \mathrm{e}-6$ & $3.33 \mathrm{e}-7$ & $8.13 \mathrm{e}-8$ & $2.00 \mathrm{e}-8$ & $4.87 \mathrm{e}-9$ & $\approx 2.02(1.50)$ \\
\hline
\end{tabular}

\subsection{Numerical results for mixed boundary conditions}

Last we illustrate the reconstruction technique for the mixed boundary condition ( $c f$. Sect. 5). In Tables 10 and 11, we present numerical results for example (c) with $q=x(1-x)$ and ${ }_{0}^{R} D_{x}^{\alpha-1} u(0)=0$ and $u(1)=0$, for three different $\alpha$ values. We observe a convergence rate of the order $O\left(h^{\min \left(2, \alpha+\ell_{n}(\beta, \gamma)\right)}\right)$ in the $L^{2}(D)$ norm, $O\left(h^{\min \left(2, \ell_{n}(\beta, \gamma)\right)-\alpha / 2}\right)$ in the $\widetilde{H}^{\alpha / 2}(D)$-norm and $O\left(h^{\min \left(2, \alpha+\ell_{n}(\beta, \gamma)\right)-1 / 2}\right)$ in the $L^{\infty}(D)$-norm for all $\alpha \in(3 / 2,2)$, which confirms Theorem 5.4. Like before, the approximate singularity strength $\mu_{h}$ achieves a second-order convergence even for $\alpha$ close to 1.5 ( $c f$. Tab. 11), which remains to be theoretically justified.

\section{CONCLUSION}

In this work, we have developed and analyzed a new finite element technique for approximating boundary value problems with a Riemann-Liouville fractional derivative in the leading term. It relies on splitting the solution into a regular part and a singular part, where the regular part lies in $H^{\alpha+\ell(\beta, \gamma)}(D) \cap \widetilde{H}^{\alpha / 2}(D)$. We derived a new variational formulation for the regular part, and showed its well-posedness and enhanced regularity. Further, a Galerkin finite element approximation for the regular part and a reconstruction formula for the singular part have been proposed. The stability of the discrete variational formulation, and error estimates of the regular part in $\widetilde{H}^{\alpha / 2}(D), L^{2}(D)$, and $L^{\infty}(D)$-norms, and the reconstructed singularity strength were established, which are higher than that for the standard Galerkin FEM approximation. The idea can be extended to other type of boundary conditions, and has also been illustrated on the mixed boundary condition.

Numerical experiments with smooth and nonsmooth source terms fully confirmed the convergence of the numerical scheme. Numerically, the $\widetilde{H}^{\alpha / 2}(D)$-estimate agree excellently with the theoretical ones. However, the $L^{2}(D)$-estimate is suboptimal: the empirical rate is one-half order higher than the theoretical one. This suboptimality is attributed to the low regularity of the adjoint solution. This has been observed earlier in the context of Poisson's equation on L-shaped domains [4] and the standard Galerkin method for fractional boundary value problems [11]. Further, the $L^{\infty}(D)$-error exhibits a superconvergence phenomenon in case of $\alpha+\ell(\beta, \gamma)>2$. The reconstructed strength $\mu_{h}$ always achieves a second-order accuracy, irrespective of the 
fractional order $\alpha$ and the smoothness of the source term, which is better than the estimate in Theorem 4.3. Optimal convergence rates in the $L^{2}(D)$ and $L^{\infty}(D)$-norms and that for the singularity strength still await mathematical justifications, for both Dirichlet and mixed problems. Last, it is of immense interest to extend the singularity approach to the multi-dimensional case and the mixed case involving both left- and right-sided fractional derivatives, for which however the solution theory remains to be developed.

Acknowledgements. The authors are grateful to two anonymous referees for their helpful comments, which have led to an improved presentation of the paper. The research of B. Jin has been partly supported by NSF Grant DMS-1319052 and National Science Foundation of China Grant No. 11471141.

\section{REFERENCES}

[1] R.A. Adams and J.J.F. Fournier, Sobolev Spaces, 2nd edition. Elsevier/Academic Press, Amsterdam (2003).

[2] Z. Bai and H. Lu, Positive solutions for boundary value problem of nonlinear fractional differential equation. J. Math. Anal. Appl. 311 (2005) 495-505,.

[3] D.A. Benson, S.W. Wheatcraft and M.M. Meerschaert, The fractional-order governing equation of Lévy motion. Water Resour. Res. 36 (2000) 1413-1424.

[4] Z. Cai and S. Kim, A finite element method using singular functions for the Poisson equation: corner singularities. SIAM J. Numer. Anal. 39 (2001) 286-299.

[5] D. del-Castillo-Negrete, B.A. Carreras and V.E. Lynch, Front dynamics in reaction-diffusion systems with Levy flights. Phys. Rev. Lett. 91 (2003) 018302.

[6] D. del-Castillo-Negrete, B.A. Carreras and V.E. Lynch, Nondiffusive transport in plasma turbulence: a fractional diffusion approach. Phys. Rev. Lett. 94 (2005) 065003.

[7] W.H. Deng and J.S. Hesthaven, Local discontinuous Galerkin methods for fractional diffusion equations. ESAIM: M2AN 47 (2013) 1845-1864.

[8] J. Douglas Jr. and T. Dupont, Galerkin approximations for the two point boundary problem using continuous, piecewise polynomial spaces. Numer. Math. 22 (1974) 99-109.

[9] A. Ern and J.-L. Guermond, Theory and Practice of Finite Elements. Springer-Verlag, New York (2004).

[10] V.J. Ervin and J.P. Roop, Variational formulation for the stationary fractional advection dispersion equation. Numer. Methods Partial Differ. Eqs. 22 (2006) 558-576.

[11] B. Jin, R. Lazarov, J. Pasciak and W. Rundell, Variational formulation of problems involving fractional order differential operators. To appear in Math. Comput. (2015).

[12] B. Jin, R. Lazarov, J. Pasciak and Z. Zhou, Error analysis of a finite element method for the space-fractional parabolic equation. SIAM J. Numer. Anal. 52 (2014) 2272-2294.

[13] A. Kilbas, H. Srivastava and J. Trujillo, Theory and Applications of Fractional Differential Equations. Elsevier, Amsterdam (2006).

[14] R. Metzler and J. Klafter, The random walk's guide to anomalous diffusion: a fractional dynamics approach. Phys. Rep. 339 (2000) $1-77$.

[15] S.G. Samko, A.A. Kilbas and O.I. Marichev. Fractional Integrals and Derivatives. Gordon and Breach, Yverdon (1993).

[16] A.H. Schatz, An observation concerning Ritz-Galerkin methods with indefinite bilinear forms. Math. Comput. 28 (1974) 959-962.

[17] E. Sousa, Finite difference approximations for a fractional advection diffusion problem. J. Comput. Phys. 228 (2009) 40384054 .

[18] C. Tadjeran and M.M. Meerschaert, A second-order accurate numerical method for the two-dimensional fractional diffusion equation. J. Comput. Phys. 220 (2007) 813-823.

[19] W. Tian, H. Zhou and W. Deng, A class of second-order finite difference approximations for solving space fractional diffusion equations. To appear in Math. Comput. (2015).

[20] H. Wang and D. Yang, Wellposedness of variable-coefficient conservative fractional elliptic differential equations. SIAM J. Numer. Anal. 51 (2013) 1088-1107.

[21] K. Yoshida, Functional Analysis, 6th edition. Springer-Verlag, Berlin (1980). 